\title{
Le mobilier métallique découvert sur le site antique de Sées « Le Grand-Herbage » (Orne)
}

\section{Mélanie Demarest}

\section{(2) OpenEdition}

\section{Journals}

Édition électronique

URL : https://journals.openedition.org/rao/371

DOI : 10.4000/rao.371

ISBN : 978-2-7535-1607-6

ISSN : 1775-3732

\section{Éditeur}

Presses universitaires de Rennes

\section{Édition imprimée}

Date de publication : 30 décembre 2007

Pagination : 169-189

ISBN : 978-2-7535-0574-2

ISSN : 0767-709X

\section{Référence électronique}

Mélanie Demarest, «Le mobilier métallique découvert sur le site antique de Sées « Le Grand-Herbage » (Orne) », Revue archéologique de l'Ouest [En ligne], 24 | 2007, mis en ligne le 30 décembre 2009, consulté le 22 août 2022. URL : http://journals.openedition.org/rao/371 ; DOI : https://doi.org/ $10.4000 /$ rao.371 


\title{
Le mobilier métallique découvert sur le site antique de Sées « Le Grand-Herbage »(Orne)
}

\author{
Mélanie Demarest*
}

\begin{abstract}
Résumé : L'établissement rural antique de Sées « Le Grand-Herbage » a livré un mobilier métallique diversifié assez proche de ce qui est couramment découvert sur ce type d'occupation. Il est présenté ici sous la forme d'un catalogue. Le mobilier se répartit en dix-sept catégories fonctionnelles différentes parmi lesquelles les éléments de construction et l'outillage dominent. Ce sont pourtant les accessoires du vêtement, peu nombreux - et en particulier une fibule en arbalète - qui retiennent l'attention. En effet, cet exemplaire d'origine germanique pose la question d'une probable réoccupation du site au Iv ${ }^{\mathrm{e}}$ siècle apr. J.-C. peut-être par un colon-soldat accompagné de sa famille.
\end{abstract}

\begin{abstract}
The metallic assemblage uncovered on the Antique farmstead of Sées "Le Grand-Herbage" (Orne)
The Antique farmstead of Sées "Le Grand-Herbage" produced a varied metallic assemblage comparable to usual inventories for this type of occupation. In the catalogue provided here, the artefacts divide up into seventeen different functional categories among which elements of building and domestic tools dominate. Nevertheless the dress accessories, although not numerous, have particular interest, especially a crossbow fibula. Indeed, this example, of Germanic origin, begs the question of a possible reoccupation of the site in IVth $c$. AD, maybe by a colonist-soldier accompanied by his family.
\end{abstract}

Mots clés : Mobilier métallique, gallo-romain, Haut-Empire, Bas-Empire, germanique, Militaire.

Key words: Metallic assemblage, Gallo-Roman, Hight-Empire, Low-Empire, Germanic fibula, Militaria.

Cet article vise à présenter le mobilier métallique découvert sur le site gallo-romain de Sées « Le Grand-Herbage » (Leclerc, 2002; Leclerc, ce volume). Il comprend 150 objets ce qui ne représente sans doute qu'une part minime du mobilier utilisé durant presque trois siècles d'occupation. Néanmoins, il s'agit d'un corpus raisonnable aux vues des ensembles couramment découverts sur les petits établissements ruraux.

L'interprétation de cet ensemble requiert certaines précautions. En effet, l'absence de contextes stratigraphiques précis interdit toute analyse spatiale et fonctionnelle. Cet article se limite donc à une présentation du mobilier métallique sous forme de catalogue. L'information, même partielle, renforce les connaissances régionales qui font particulièrement défaut à ce sujet.

*Archéologue contractuelle. (melanie.demarest@wanadoo.fr)

\section{Présentation synthétiQue du corpus}

Le mobilier se répartit en 17 grandes catégories présentées ci-dessous (fig. 1). Il comprend 120 objets en fer, 26 en alliage cuivreux et 4 en plomb.

Le statut des occupants du site est particulièrement difficile à définir. Certains objets peuvent cependant constituer quelques pistes. Une phalère ( $\mathrm{n}^{\circ} 12$, fig. 2$)$, à décor niellé, suggère la possession d'un cheval et l'utilisation d'un harnachement soigné. L'usage de nielle pourrait la rapprocher de certains exemplaires produits au I ${ }^{\text {er }}$ siècle apr. J.-C. (Rabeisen, 1990, p. 73-94), ce qui n'exclut pas pour autant une datation plus récente. Le corpus comprend aussi deux fers de javeline ( $\mathrm{n}^{\circ} 13$ et 14) de morphologies différentes (types I et II de Manning : 1985, p. 162-166); mais traduisent-ils un statut particulier ou tout simplement la pratique de la chasse? Ils proviennent vraisemblablement de deux phases d'occupation distinctes. 


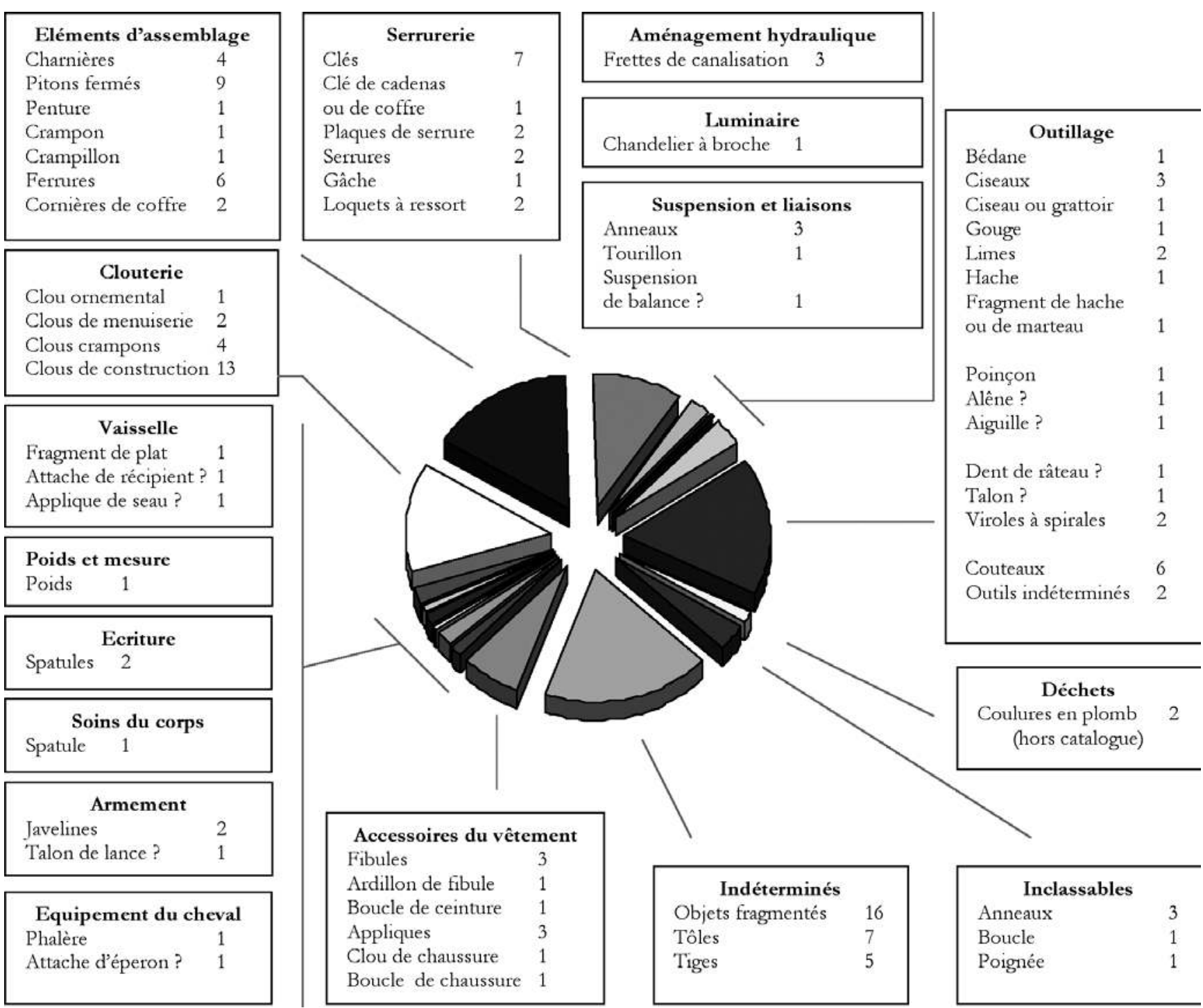

Figure 1 : Sées «Le Grand-Herbage ": répartition générale du mobilier métallique par catégories typologiques.

Figure 1: General distibution of metal assemblage by typological categories.

Parmi les accessoires du vêtement, deux fibules présentent un décor émaillé. L'une ( $\left.\mathrm{n}^{\circ} 1\right)$ appartient à un modèle produit à partir du milieu du I ${ }^{\text {er }}$ siècle apr. J.-C. et dont l'utilisation se prolonge aux $\mathrm{II}^{\mathrm{e}}$ et $\mathrm{III}^{\mathrm{e}}$ siècles. La seconde $\left(\mathrm{n}^{\circ} 2\right)$ est à rapprocher d'un style bien particulier dont la production se situe entre 135 et 170 apr. J.-C. L'une et l'autre sont diffusées et probablement fabriquées en Gaule septentrionale. Mais la fibule la plus remarquable se trouve être une pièce en arbalète $\left(\mathrm{n}^{\circ} 3\right)$, dont l'usage est attesté entre la seconde moitié du $\mathrm{III}^{\mathrm{e}}$ et le début de la seconde moitié du Iv siècle apr. J.-C. Pendant germanique de la fibule cruciforme romaine, sa diffusion se concentre au nord de la Germanie libre (Philippe, 1997, p. 9). Particulièrement rares en Gaule septentrionale, ces exemplaires sont couramment mis en relation avec le déplacement et l'implantation d'individus d'origine germanique.
Dans la région, d'autres sites ont livré du mobilier de même origine, comme Lisieux " Michelet " (Calvados), Fel (Orne) et Pouligny (Calvados) (Paillard, 1994, p. 71-72, 96), ainsi que Frénouville (Calvados : Pilet, 1980, p. 171) et Saint-Martin-de-Fontenay (Calvados : Pilet et al., 1994, p. 82). Ce phénomène est souvent attribué aux déplacements de l'armée romaine, dont les contingents sont en grande partie constitués de mercenaires «barbares " (Paillard et AlducLe Bagousse, 1996, p. 27). Cette hypothèse pourrait être relayée ici par la présence d'une boucle $\left(n^{\circ} 5\right)$, dont la forme rappelle celle de modèles découverts sur des ceinturons militaires du Bas-Empire (Oldenstein, 1976, p. 213-214, n 990 pl. 75). De même, les appliques ( ${ }^{\circ}$ 6-8) correspondent à des modèles pouvant être associés aux courroies de ceinturon militaire (Bishop 1992, p. 81-95; Oldenstein, 1976, p. 171- 
172, pl. 48). Dans la même optique, les éléments d'armement et de harnachement recueillis au Grand-Herbage peuvent aussi être liés au domaine militaire.

À la vue de ces différents éléments, l'occupation du site par un colon-soldat accompagné de sa famille est une hypothèse envisageable. Néanmoins, il est périlleux de tirer toute conclusion à partir de ces indices dont la concordance chronologique ne peut être attestée, faute de contextes stratigraphiques.

La vie quotidienne est abordée ici par différents biais comme l'écriture, les soins du corps, les poids et mesures ou la vaisselle, mais toujours de manière anecdotique. En effet, seulement huit objets se rapportent à ce domaine.

Les éléments d'assemblage et la serrurerie liés aux bâtiments sont bien représentés. Il faut noter à ce sujet la présence de deux serrures complètes, ce qui est particulièrement rare ( $\left.n^{\circ} 62-63\right)$. Quelques frettes de canalisations présument l'utilisation d'un système hydraulique composé de conduites d'eau en bois, les frettes assurant leur jonction. En revanche l'aménagement intérieur n'est évoqué que par le biais d'un chandelier à broche.

Le domaine de l'outillage occupe une bonne place au sein du corpus. Il comprend des outils d'utilisation courante. Certains sont plus probablement utilisés pour le travail du bois (divers ciseaux, gouge, hache...); d'autres sont plus à associer au domaine de la vie quotidienne comme les couteaux.

Globalement, le mobilier découvert sur ce site présente une distribution fonctionnelle assez commune avec une forte représentation d'éléments associés aux domaines de la construction et de l'outillage. Les autres catégories comme les accessoires du vêtement sont généralement moins bien représentées. C'est pourtant ce dernier domaine qui offre le plus d'informations concernant l'occupation du site. En effet, la fibule en arbalète $n^{\circ} 3$ offre un indice supplémentaire au sujet d'une réoccupation du site au IV siècle apr. J.-C. Sa présence, associée à quelques objets pouvant se rapprocher du domaine militaire, offre quelques pistes quant au statut éventuel des derniers occupants du site. Même si pour les raisons précisées plus haut, l'hypothèse d'un colon-soldat originaire d'OutreRhin reste fragile, ce mobilier fournit malgré tout une trace supplémentaire de la présence d'une population d'origine germanique dans la région au Bas-Empire.

\section{Catalogue}

\section{La vie domestique}

Certains mobiliers permettent d'appréhender la vie quotidienne des occupants d'un site. Les accessoires du vêtement, l'équipement du cheval et l'armement sont plus particulièrement liés à leur statut social. Les thèmes de l'hygiène cor- porelle, de l'écriture, des poids et mesures et de la vaisselle permettent de dresser une image de leur quotidien.

\section{Accessoires du vêtement et parure (fig. 2)}

001. Fibule (Bas-Empire - Phase 2; abandon d'un bac de préparation de la chaux).

Fibule (alliage base cuivre) présentant une charnière à goupille formée par le repli de l'arc sur lui-même. L'arc, épais, de forme triangulaire, est orné de six loges triangulaires ayant perdu l'émail qu'elles contenaient. Le pied est séparé de l'arc par une bande ciselée; il s'achève par une petite protubérance moulurée. L'ardillon est absent. L. $4,4 \mathrm{~cm} ; 1.3,7 \mathrm{~cm}$.

Cette fibule est à rapprocher du type $26 \mathrm{~b} 1 \mathrm{de} \mathrm{M}$. Feugère (1985, p. 356). Son décor relève d'une technique maîtrisée sous Néron, soit à partir du milieu du $\mathrm{I}^{\mathrm{er}}$ siècle apr. J.-C. (ibid., 1985, p. 364; Philippe, 2000, p. 146). Sa période d'utilisation se prolonge aux $\mathrm{II}^{\mathrm{e}}$ et $\mathrm{III}^{\mathrm{e}}$ siècles. Une fibule similaire à été découverte en Haute-Normandie (Dollfus, 1973, p. 222, n 495).

002. Fibule (Bas-Empire - Niveau de circulation F124/ F125).

Fibule (alliage base cuivre) à charnière à plaquette. L'arc est formé par deux disques percés présentant un émaillage polychrome rayonnant où on distingue encore le bleu. L'espace situé entre les disques possède quatre loges triangulaires dont une est emplie d'émail rouge. Des lignes de trois ocelles sont disposées de part et d'autre des triangles et entre les cercles. La tête de l'arc et le pied représentent une tête de reptile stylisée. L. 5,9 cm; $1.3,6 \mathrm{~cm}$; diam. des disques $1,75 \mathrm{~cm}$.

Cette fibule appartient au type 26c4 (Philippe, 2000, p. 149-150) et est plus particulièrement à rapprocher du style X2a variante D (ibid., p. 156-157). La largeur des disques diffère d'un millimètre de celle des productions classiques de ce type; néanmoins, ces modèles sont suffisamment proches et rares pour émettre l'hypothèse d'un même atelier ou d'une même aire de production. Ces objets se répartissent selon deux aires : l'une dans les départements du Nord et du Pas-de-Calais, l'autre en Île-de-France (ibid., p. 157). Selon J. Philippe, les exemplaires découverts hors de ces zones sont trop rares et trop dispersés pour correspondre à une diffusion commerciale et seraient plutôt des apports individuels.

003. Fibule en forme d'arbalète (Habitat du Bas-Empire).

La tête de la fibule (alliage base cuivre) est formée par un ressort bilatéral à corde interne dans lequel s'insère une goupille qui traverse la tête perforée de l'arc. Deux bulbes moulurés sont fixés de part et d'autre du ressort. L’arc, de profil semi-circulaire, présente un corps à trois pans faiblement marqués, encadré par un décor incisé au niveau de sa tête et à sa jonction avec le pied. Ce dernier montre une 


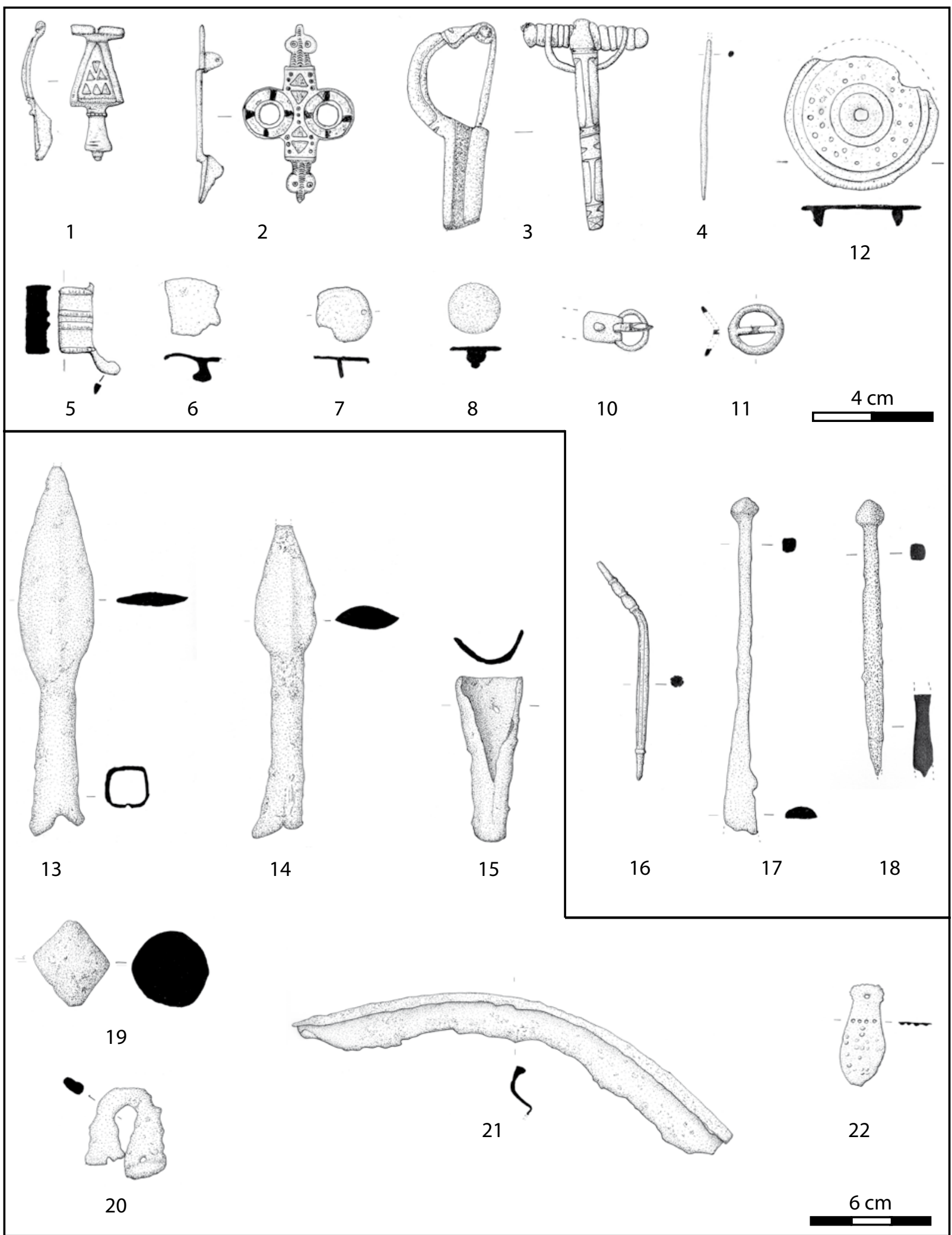

Figure 2 : Éléments de parures (1-10), de harnachement (11-12), d'armement (13-15); instruments de toilette (16), instruments d'écriture (17-18); poids (19); fragments de vaisselle (20-22) - détails dans le texte.

Figure 2: Jewellery (1-10), horse gear (11-12), weaponry (13-15); toilet equipment (16), writing (17-18); weight (19); fragments of tableware (20-22) - see text for details. 
ornementation similaire. L. tête $4,1 \mathrm{~cm}$; L. fibule $7 \mathrm{~cm}$; H. 2,9 cm; L. pied 3,4 cm. Si l'on en croit l'encoche disposée au sommet de la tête de l'arc et au vu d'exemplaires proches, cette fibule devait comporter un bouton sommital (Koller et Herzog, 1998, p. 129; collectif 1983, p. 307-308, fig. 267f).

La pièce se rapproche du type 6 de Keller (Philippe, 1997, p. 9). Son usage est attesté entre la seconde moitié du III $^{\text {e }}$ siècle et le début de la seconde moitié du IV apr. J.-C. Ce modèle a probablement été produit dans la région de l'actuelle Basse-Saxe entre l'Elbe et la Weser, et sa diffusion se concentre au nord de la Germanie libre (ibid.). Sa présence en Gaule septentrionale est marginale et semble devoir être mise en relation avec le déplacement et l'implantation d'individus d'origine germanique. Ce modèle est associé au costume féminin (ibid.; collectif, 1983, p. 306-308). En effet, celui-ci est porteur de tradition dans ces sociétés et ces fibules n'ont pas de valeur intrinsèque (Kazanski, 1996, p. 20).

\section{Ardillon.}

Tige (alliage base cuivre) de section circulaire effilée vers une extrémité et brisée au niveau de la seconde. L. inc. $5,4 \mathrm{~cm}$; D. $0,2 \mathrm{~cm}$. Cet ardillon est trop long pour correspondre aux fibules étudiées; il appartient donc à un autre individu.

\section{Boucle de ceinturon.}

Élément (alliage base cuivre) présentant un corps de section et de forme quadrangulaire orné de bandes longitudinales et de stries sur une face. L'anneau de la boucle est brisé. H. $2 \mathrm{~cm}$; L. inc. $3 \mathrm{~cm}$; ép. $0,8 \mathrm{~cm}$. Une boucle découverte à Miltenberg présente une facture assez proche. Il s'agit d'un modèle associé aux ceinturons militaires du Bas-Empire (Oldenstein, 1976, p. 213, 214, n 990 pl. 75).

\section{Applique.}

Tôle (alliage base cuivre) de forme quadrangulaire dont le bord conservé se courbe vers le tenon situé au revers. L. inc. $1,9 \mathrm{~cm} ; 1.1,7 \mathrm{~cm}$; Ht. $0,8 \mathrm{~cm}$; ép. $0,1 \mathrm{~cm}$.

\section{Applique.}

Élément (alliage base cuivre) à tête circulaire plate. Il est orné d'une moulure périphérique et son rebord se courbe vers la tige. Une perforation carrée en périphérie signale l'utilisation d'un clou pour sa fixation. Le tenon situé au revers se compose d'une mince tige de section carrée non effilée. Le pourtour est partiellement tronqué. L. 2,5 cm; Ht. $0,7 \mathrm{~cm}$.

\section{Applique.}

Élément (alliage base cuivre) présentant une tête circulaire plate portant un tenon au revers. L. $1,75 \mathrm{~cm}$; Ht. $0,6 \mathrm{~cm}$.

De tels exemplaires peuvent être utilisés pour l'habillement ou le harnachement (Crummy, 1983, p. 115-118). Ils correspondent à un modèle utilisé pour orner des lanières de cuir suspendues sur les ceinturons militaires et certains harnachements (Bishop, 1992, p. 81-95; Oldensten, 1976, p. 171-172, pl. 48).

\section{Clou de chaussure (non dessiné).}

Clou (fer) à tête hémisphérique pleine partiellement conservée. Ht. 1,6 cm;1. 1,1 cm. Ce type de clou est utilisé sur les chaussures (Guillaumet et Nilesse, 2000, p. 252).

\section{Boucle de chaussure?}

Boucle (alliage base cuivre) hémisphérique prolongée par une tôle repliée sur elle-même et fixée par un rivet. La surface est étamée et l'ardillon présente une face à plusieurs pans et une bande moulurée. L. totale inc. $2,1 \mathrm{~cm}$; L. boucle $1,5 \mathrm{~cm}$; Ht. $1,1 \mathrm{~cm}$; ép. $0,2 \mathrm{~cm}$. Ses dimensions réduites la rapprochent de certains modèles de boucles de chaussure découvertes dans des contextes médiévaux.

\section{Équipement du cheval (fig. 2)}

\section{Boucle d'attache d'éperon.}

Anneau (alliage base cuivre) de section et forme circulaires présentant une traverse médiane. Cette dernière possède un léger creux destiné à caler l'ardillon (absent). D. ext. 1,7 cm; ép. $0,2 \mathrm{~cm}$.

Cette forme de boucle est utilisée pour attacher les éperons au bas Moyen-Âge (Egan et Pritchard, 1991, p. 65-66, $n^{\circ}$ 214; Halbout et al., 1987, p. 185).

\section{Phalère.}

Phalère (alliage base cuivre) composée d'une plaque de forme circulaire et de section quadrangulaire. Elle est ornée de stries fines et rayonnantes sur son pourtour et d'incisions concentriques. La bande centrale est parsemée de creux circulaires, mais la corrosion ne permet pas de distinguer s'ils contenaient de l'émail ou du nielle. La surface présente encore quelques traces d'étamage. La partie médiane est perforée. L'autre face comporte quatre appendices dont l'un est brisé. Le pourtour est légèrement tronqué. D. $5 \mathrm{~cm}$; ép. $0,1 \mathrm{~cm}$. Il s'agit d'un médaillon de phalère à deux passants destiné à permettre la jonction entre les lanières de cuir d'un harnachement. Ces médaillons étaient des pièces à la fois fonctionnelles et décoratives. Des exemplaires niellés sont produits au $\mathrm{I}^{\text {er }}$ siècle apr. J.-C. (Rabeisen 1990, p. 73-94); néanmoins, cet exemplaire peut être plus récent. 


\section{Armement (fig. 2)}

013. Fer de javeline (Haut-Empire - Niveau de circulation F124/F125).

Élément (fer) à emmanchement à douille fermée et à lame en forme de "feuille de saule " de section lenticulaire. L'extrémité de la douille présente une forme sub-quadrangulaire et est brisée. La surface originelle est altérée par la corrosion. L. inc. douille : 7,8 cm; l. et ht. douille : $2,3 \mathrm{x}$ $2,1 \mathrm{~cm}$, L. lame : $10,5 \mathrm{~cm} ; 1 .: 3,6 \mathrm{~cm}$; ép. : 0,7 cm.

014. Fer de javeline (Haut-Empire - Dépotoir 2).

Élément de morphologie massive (fer) présentant un emmanchement à douille fermée et une lame en forme de "feuille de saule ", de section ovale. La lame est légèrement asymétrique et désaxée par rapport à la douille. L'épaulement formé entre lame et douille est nettement moins marqué sur un côté (usure?). La lame est nettement plus petite que la douille. Celle-ci est robuste et son extrémité brisée présente une section sub-rectangulaire. L. inc. douille $8,5 \mathrm{~cm}$; L. lame $6,5 \mathrm{~cm} ; 1.2,9 \mathrm{~cm}$; ép. $1,1 \mathrm{~cm}$; l. et ht. douille 2,4 $\mathrm{x} 1,5 \mathrm{~cm}$.

Le fer $\mathrm{n}^{\circ} 13$ se rapproche des exemplaires découverts à Kingsholm et Hod Hill (Manning 1985, p. 160-162, 165$\left.166, \mathrm{n}^{\circ} 32,82,86\right)$. Ses dimensions sont légèrement supérieures à celles de ces pièces (Longueurs de lame 8 à $10 \mathrm{~cm}$ et largeurs 2 à $3 \mathrm{~cm}$ : ibid., p. 165), mais la forme générale reste la même. Toutefois, il faut noter qu'aucun exemplaire de ces sites ne présente de douille quadrangulaire; néanmoins, notre $\mathrm{n}^{\circ} 13$ pourrait se rapprocher du type II de W. H. Manning (ibid., p. 165-166). Le n 14 serait plutôt à comparer au groupe I de W. H. Manning (ibid., p. 162-165) et notamment avec l'exemplaire 58 (ibid., pl. 77), qui présente lui aussi une douille relativement longue par rapport à la lame. La fonction des fers de lance de ces deux groupes reste en suspens, leur forme ne permet pas de déterminer à elle seule leur utilisation précise; un usage comme pique est aussi possible.

015. Talon de lance? (Haut-Empire - Niveau de circulation F124/F125).

Tôle (fer) martelée en douille ouverte et évasée d'un côté; l'extrémité opposée est fermée. Une perforation rectangulaire est visible sur la partie supérieure. L. $8,2 \mathrm{~cm} ; 1.1,4$ à $3,3 \mathrm{~cm}$; ép. tôle $0,2 \mathrm{~cm}$; ép. objet $2,2 \mathrm{~cm}$. Ce type d'objet est couramment interprété comme talon destiné entre autres à protéger l'extrémité du manche en bois d'outils ou de lances (Manning, 1985, p. 140-141, pl. 66). Néanmoins, celuici semble bien large et peu appointé pour une utilisation sur une hampe de lance (cf. Sievers, 1996, p. 74-75).
Hygiene et soins du corps (fig. 2)

\section{Spatule?}

Tige (alliage base cuivre) de section sub-circulaire dont le corps renflé au centre s'effile vers chaque extrémité appointée. Le corps présente un décor de bandes longitudinales. L'extrémité la plus épaisse présente une succession de moulures puis s'effile en pointe; la seconde est séparée du corps par une fine bande transversale. La tige est tordue sur la seconde extrémité. L. 7,9 cm; D. max. 0,4 cm. Il s'agit d'un instrument probablement de type spatule, utilisé pour les soins du corps et pour certains usages médicaux (Fauduet 1992, p. 131-132, n 947).

\section{Écriture (fig. 2)}

\section{Spatule à cire.}

Élément (fer) constitué d'une tige de section hémisphérique achevée par un bouton d'un côté et martelée en spatule de l'autre. L. inc. $11,15 \mathrm{~cm} ; 1.1,5 \mathrm{~cm}$; ép. $0,5-0,3 \mathrm{~cm}$. Le bouton qui orne sa partie de préhension n'a pas été utilisé comme surface de frappe. Cet objet a pu servir de spatule destinée à effacer les tablettes de cire utilisées comme support d'écriture.

\section{Spatule à cire?}

Tige (fer) de section carrée, surmontée d'un bulbe biconique d'un côté et aplatie au niveau de la seconde extrémité (brisée). L. 9,5 cm; 1. bulbe $0,9 \mathrm{~cm} ; 1$. spatule $0,5 \mathrm{~cm}$. Le bulbe ne semble pas avoir été utilisé comme surface de frappe. Comme le précédent, cet outil a pu être utilisé comme spatule à cire.

Les spatules sont couramment interprétées comme ustensiles pour le travail des matières plastiques. L'extrémité bouletée de ces deux exemplaires semble témoigner d'une certaine finition dans la conception. Ce détail et l'étroitesse des spatules tendent à suggérer une utilisation comme spatule à cire (cf. Feugère et Lambert, 2004, p. 32).

\section{Poids et mesures (fig. 2)}

\section{Poids.}

Élément (plomb) de forme biconique; un des sommets présente une petite tige en fer brisée. Ht. $4 \mathrm{~cm}$; D. $3,7 \mathrm{~cm}$. Cette forme est souvent interprétée comme peson de balance romaine en raison des découvertes de balances complètes qui attestent son utilisation pour cette fonction (Mitard, 1993, p. 386 et 388).

\section{Vaisselle (fig. 2)}

\section{Attache de récipient?}

Tige (fer) de section rectangulaire, courbée symétriquement en « $U$ ». Ses extrémités perforées sont martelées et éti- 
rées dans le sens de la largeur. Une des extrémités est tordue et la seconde est brisée. L. $4,3 \mathrm{~cm} ; 1.3,9 \mathrm{~cm} ; 1$. tige $2 \mathrm{~cm}$; ép. $0,7 \mathrm{~cm}$. Sa forme suggère une utilisation comme élément de suspension. Il peut s'agir d'une attache de récipient en bois, par exemple (Eneau, 2002, p. 35, n 72 ; Jackson, 1990, p. 8, no 9).

\section{Fragment de plat.}

Tôle (alliage base cuivre); plus épaisse et raplatie sur un bord, elle se courbe légèrement puis à angle droit vers le second bord, brisé. Sa forme générale évoque un rebord de plat. Ep. 0,2 à $0,3 \mathrm{~cm}$.

\section{Applique de seau?}

Tôle (alliage base cuivre) de forme lancéolée et dont l'extrémité supérieure présente une perforation et un pourtour dentelé. L'avers a été poinçonné de manière à fournir des points en relief sur la face. La première ligne fait exception en étant poinçonnée côté face. Par ailleurs, l'avers présente une substance gris clair sur une partie de sa surface (résidu de brasure?). L. $5,1 \mathrm{~cm} ; 1.2,3 \mathrm{~cm}$; ép. $0,1 \mathrm{~cm}$.

\section{La construction}

Le domaine de la construction renvoie à l'ensemble de la quincaillerie destinée à permettre l'assemblage des différentes pièces d'huisserie et aux divers aménagements associés au fonctionnement des bâtiments.

\section{Clouterie}

Il semble fort probable que le mobilier qui nous a été soumis ait subit un premier tri à l'œil durant la fouille et que seuls quelques clous aient été retenus pour étude. Ces derniers n’ont pas été dessinés; ils ne présentent pas de particularité et entrent dans les groupes les plus courants : clous ornementaux, clous de menuiserie, clous crampons et clous de construction (Guillaumet, 2003, p. 132-133).

\section{Clou ornemental (non dessiné).}

Clou (alliage base cuivre) à tête hémisphérique creuse. L. $1,8 \mathrm{~cm} ; 1.1,5 \mathrm{~cm}$; Ht. $0,95 \mathrm{~cm}$. De tels éléments ont souvent un usage décoratif en ameublement. Les plus courts servent à fixer des placages de bois, de cuir, d'os ou de tissu (Romeuf, 2001 [T. 1], p. 195). Toutefois, ils peuvent aussi être utilisés pour l'habillement, l'équipement militaire ou le harnachement (Crummy, 1983, p. 115-118).

\section{Clou (non dessiné).}

Tige massive (fer) de section rectangulaire, effilée vers une extrémité. Elle se courbe puis se coude au niveau de l'extrémité la plus fine. L. $5,3 \mathrm{~cm}$; Ht. 3,3 cm; 1. x ép. 1,1 x
$1,05 \mathrm{~cm}$. Ce clou a du être réutilisé en crochet (Halbout $e t$ al., 1987, p. 180).

025. Clou (non dessiné).

Clou (fer) de type " tête d'homme ", de section carrée effilée au niveau d'une extrémité. Les extrémités sont coudées lui conférant une forme en « $U$ ». L. 4,6 cm; Ht. $3,3 \mathrm{~cm}$; l. x ép. $0,75 \times 0,8 \mathrm{~cm}$.

026. Clous crampon (non dessinés).

Quatre clous à tête en " $T$ » dont la surface supérieure est bombée. L. 10,2 à 13,2 cm; l. tête 2,3 à 3,1 cm. Ce genre de clou appartient au « type 3 " proposé par W. H. Manning (1985, p. 31-133, fig. $\left.32 \mathrm{n}^{\circ} 3\right)$.

027. Clous de construction (non dessinés).

Treize clous à tête circulaire et carrée. L. 10,7 à $15 \mathrm{~cm}$; L. tête 2,7 à $3 \mathrm{~cm}$; ép. 0,8 à $1,1 \mathrm{~cm}$.

\section{Éléments d'assemblage (fig. 3 et 4)}

\section{Charnière}

Tôle (fer) repliée en cylindre et prolongée à chaque extrémité par une patte de fixation de section rectangulaire. Les pattes sont brisées. L. inc. $6,4 \mathrm{~cm}$; 1 . ext. nœud $2 \mathrm{~cm}$. Il s'agit de la partie de charnière formant le nœud qui permet au gond de tourner (Halbout et al., 1987, p. 58).

\section{Charnière à encastrer}

Charnière (fer) constituée d'une tige plate assemblée à une seconde tige brisée par une partie renflée dans laquelle passe un axe. La tige est tronquée et présente un clou de fixation. L. inc. $6,5 \mathrm{~cm} ; 1.2 \mathrm{~cm}$; Ht. $1,9 \mathrm{~cm}$; ép. $0,8 \mathrm{~cm}$. Ce système de charnière permet au battant de pivoter (Guillaumet 2003, p. 130).

\section{Charnière à fiches}

Charnière (fer) formée de trois ailes articulées autour d'un rivet. Chaque aile est constituée par une tige de section rectangulaire repliée symétriquement, formant ainsi deux fiches appointées. La seule fiche entièrement conservée est légèrement coudée sur son extrémité. Cet objet est partiellement brisé et légèrement altéré par la corrosion. L. $7 \mathrm{~cm}$; 1. $2,8 \mathrm{~cm}$.

\section{Charnière à fiches}

Charnière (fer) formée de trois ailes articulées autour d'un rivet. Chaque aile est constituée par une tige de section hémisphérique repliée symétriquement, formant ainsi deux fiches appointées. Une seule aile est entièrement conservée : ses deux extrémités sont courbées. Les deux ailes sont brisées. L. 5,7 cm;1. 4,2 cm. Ce système de charnière à fiches 


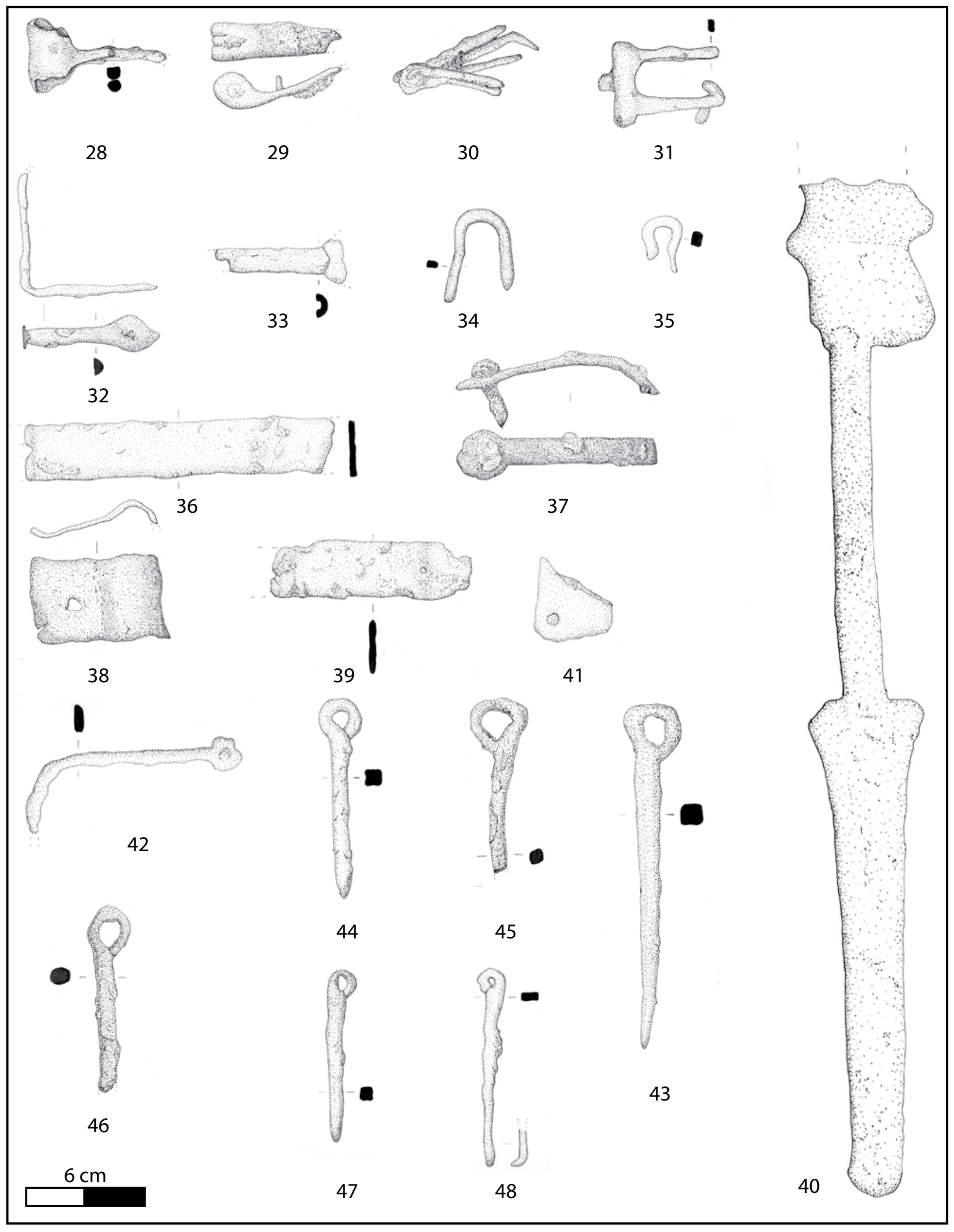

Figure 3 : éléments d'assemblage (28-31).

Figure 3: Joinery equipment (28-31). 
permet la rotation par la fixation des ailes dans le montant et le battant : les deux ailes extérieures sont fixées d'un côté et l'aile centrale de l'autre. Elle est utilisée pour les coffres et autres meubles (Guillaumet, 2003, p. 131).

\section{Cornière de coffre}

Tige (fer) de section quadrangulaire coudée en équerre et présentant une extrémité lancéolée perforée en son centre. La seconde extrémité est brisée. L. $6,8 \mathrm{~cm} ; 1.1,7 \mathrm{~cm}$; ép. $0,4 \mathrm{~cm}$.

\section{Cornière de coffre?}

Élément (fer) constitué d'une tige à section en « $U$ » martelée et aplatie au niveau d'une extrémité. Cette dernière est incomplète et prend une forme semi-circulaire perforée en son centre. La seconde extrémité est brisée. L. inc. $6,4 \mathrm{~cm}$; 1. $2,2 \mathrm{~cm}$; ép. $0,6 \mathrm{~cm}$.

\section{Crampillon}

Élément (fer) de section rectangulaire replié en forme de " $U$ ». Les extrémités sont effilées et l'une d'entre elles est brisée et tordue. L. 4,7 cm; 1. 3,6 cm ; ép. 0,6 à 0,4 cm. Il s'agit d'un type de crampon (Eneau 2002, p. 16-17, n 80 à 85).

\section{Crampon}

Cet élément (fer) de section carrée présente deux branches en lacet formant une boucle. L'une est brisée et la seconde est aplatie vers l'extrémité. L. $3 \mathrm{~cm} ; 1.2 \mathrm{~cm}$; ép. $0,65 \mathrm{~cm}$. Ce type d'élément peut avoir été utilisé pour suspendre un anneau ou comme point d'ancrage à rôle multiple (Halbout et al., 1987, p. 177).

\section{Ferrure?}

Tige (fer) de section rectangulaire, légèrement courbée au niveau d'une extrémité et perforée. Aucune extrémité n'est conservée. L. inc. $15,7 \mathrm{~cm} ; 1.2,9 \mathrm{~cm}$; ép. 0,4 cm.

037. Ferrure (Haut-Empire - Niveau de circulation F124/ F125)

Tige (fer) de section rectangulaire et courbée sur un quart de sa longueur. Elle présente une extrémité de forme arrondie traversée par un clou en son centre. La seconde extrémité est brisée. L. inc. $9,7 \mathrm{~cm} ; 1.2,5 \mathrm{~cm}$; ép. 0,5 cm. La courbure de cet élément n'est pas assez marquée pour permettre une utilisation comme poignée. Il s'agit vraisemblablement d'un élément d'assemblage de type ferrure (Eneau, 2002, pl. 25).

\section{Ferrure}

Plaque (fer) de section rectangulaire présentant un profil courbe au niveau de son extrémité brisée. La partie rectili- gne comporte une perforation destinée à assurer sa fixation. L. inc. $6,5 \mathrm{~cm} ; 1.4,4 \mathrm{~cm}$; ép. $0,3 \mathrm{~cm}$.

\section{Ferrure?}

Tôle (fer) de section rectangulaire, présentant une soie au niveau d'une extrémité et brisée de l'autre. Elle comporte deux perforations destinées à assurer sa fixation. L. inc. $10 \mathrm{~cm} ; 1.2,8 \mathrm{~cm}$; ép. $0,2 \mathrm{~cm}$. Cet objet a pu être utilisé comme élément d'assemblage de type ferrure; la soie peut avoir servi de fixation supplémentaire.

\section{Ferrure}

Élément (fer) constitué de trois parties. La partie centrale, légèrement tordue, est constituée d'une tige de section rectangulaire; elle est martelée en plaque de forme triangulaire à extrémité arrondie au niveau d'une extrémité et se prolonge de l'autre côté par une plaque à bords concaves, évasée et de profil courbe. Cette partie est tronquée. L. inc. $51,2 \mathrm{~cm}$; $1.7 \mathrm{~cm}$; ép. $0,7 \mathrm{~cm}$. La morphologie et la robustesse de cet objet suggèrent une utilisation comme ferrure.

\section{Ferrure?}

Plaque (fer) de section rectangulaire brisée. Un des angles présente un clou de fixation. L. inc. $4,1 \mathrm{~cm} ; 1.4,15 \mathrm{~cm}$; ép. $0,5 \mathrm{~cm}$.

042. Penture? (Haut-Empire - Niveau de circulation F124/ F125)

Tige (fer) de section rectangulaire, coudée et brisée sur une extrémité et achevée de l'autre côté par un nœud destiné à permettre sa rotation. Cette partie est corrodée et partiellement brisée. L. inc. $9,8 \mathrm{~cm} ; 1.1,8 \mathrm{~cm}$; ép. 0,4 cm.

\section{Piton fermé}

Tige (fer) de section carrée effilée d'un côté et repliée en boucle sur la seconde extrémité. La boucle est décentrée. L. $17,6 \mathrm{~cm}$; 1 . boucle $2,7 \mathrm{~cm} ; 1$. tige $1,1 \mathrm{~cm}$; ép. $1 \mathrm{~cm}$.

044. Piton fermé (Haut-Empire - Niveau de circulation F124/F125)

Tige (fer) de section quadrangulaire étirée et repliée en boucle au niveau d'une extrémité. La seconde extrémité est brisée. L. inc. $9,95 \mathrm{~cm} ; 1.2,1 \mathrm{~cm}$.

\section{Piton fermé}

Tige (fer) de section carrée, martelée et étirée en boucle au niveau d'une de ses extrémités. La tige tordue s'amincit vers la pointe. L. $8,7 \mathrm{~cm} ; 1.2,9 \mathrm{~cm}$. 


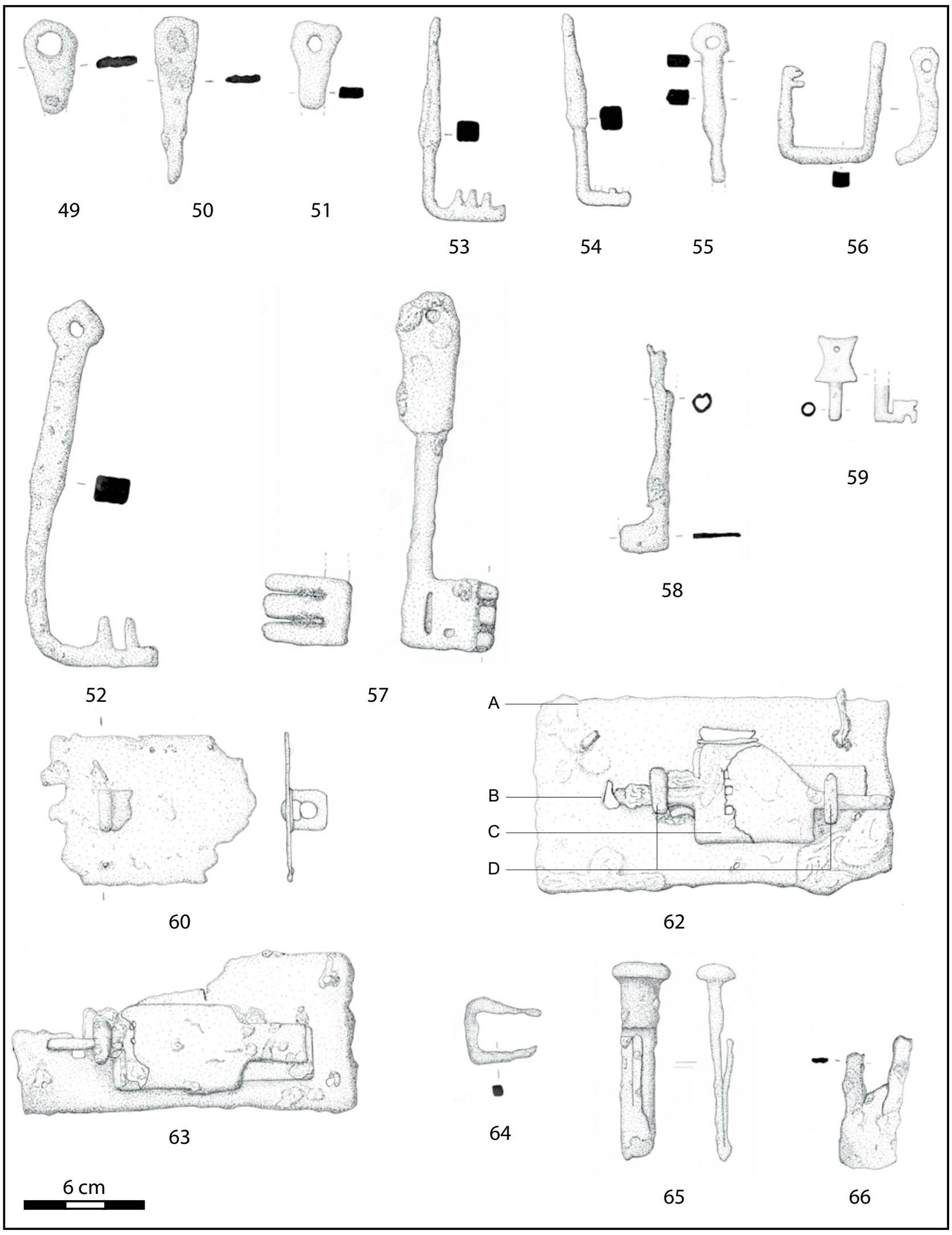

Figure 4 : Éléments d'assemblage, suite (49-51); pièces de serurerie (52-66).

Figure 4: Further joinery equipment (49-51); lock components (52-66). 
046. Piton fermé (Haut-Empire - Niveau de circulation F124/F125)

Tige (fer) de section quadrangulaire, brisée sur l'extrémité et martelée en boucle fermée sur la seconde. L. inc. $9,3 \mathrm{~cm}$; 1. tige $1 \mathrm{~cm}$; ép. tige $0,9 \mathrm{~cm} ; 1$. boucle $2,1 \mathrm{~cm}$.

047. Piton fermé (Haut-Empire - Niveau de circulation F124/F125)

Tige (fer) de section carrée, effilée vers une extrémité et étirée puis repliée en boucle de l'autre. L. $8,7 \mathrm{~cm}$; l. boucle $1,9 \mathrm{~cm}$; ép. $0,5 \mathrm{~cm}$.

\section{Piton fermé}

Tige (fer) méplate, effilée vers une extrémité et étirée puis repliée en boucle de l'autre. La partie effilée est coudée au niveau de son extrémité. Le profil est légèrement courbe. L. $9,8 \mathrm{~cm}$; 1 . boucle $1,3 \mathrm{~cm}$; ép. $0,4 \mathrm{~cm}$.

\section{Piton fermé}

Élément (fer) composé d'une plaque percée pour former un anneau sur l'extrémité et martelée en tôle de section rectangulaire effilée vers la seconde. Cette seconde partie est tordue et brisée. L. inc. $4,8 \mathrm{~cm} ; 1.2,7 \mathrm{~cm}$; ép. $0,6 \mathrm{~cm}$.

050. Piton fermé (Haut-Empire - Niveau de circulation F124/F125)

Élément (fer) présentant une section rectangulaire. Il se compose d'une plaque repercée en anneau au niveau d'une extrémité puis martelée et effilée vers la seconde, brisée. L. inc. $4,3 \mathrm{~cm} ; 1.2,3 \mathrm{~cm}$; ép. $0,6 \mathrm{~cm}$.

\section{Piton fermé}

Élément (fer) de forme triangulaire et de section rectangulaire. L. $8,5 \mathrm{~cm} ; 1.2,2 \mathrm{~cm}$; ép. $0,3 \mathrm{~cm}$.

Les exemplaires $\mathrm{n}^{\circ} 41$ à 46 sont des pitons fermés par enroulement et les $\mathrm{n}^{\circ} 47$ à 49 des pitons fermés par percement (Guillaumet 2003, p. 38). Ils peuvent être utilisés comme partie femelle d'une charnière, pour laisser coulisser un lien ou pour permettre la suspension d'un autre objet.

\section{Serrurerie (fig. 4)}

052. Clé (Haut-Empire - Dépotoir 2)

Clé (fer) à embase pyramidale et dont la tige coudée comporte deux dents. L. $18,3 \mathrm{~cm} ; 1.6,1 \mathrm{~cm}$.

053. Clé (Haut-Empire - Niveau de circulation F124/ F125)

Clé (fer) à embase pyramidale et dont la tige coudée comporte trois dents. L. $10,1 \mathrm{~cm} ; 1.4,2 \mathrm{~cm}$.
054. Clé (Haut-Empire - Niveau de circulation F124/ F125)

Clé (fer) à embase pyramidale et dont la tige coudée comporte trois dents, toutes tronquées. L. $9,4 \mathrm{~cm} ; 1.2,7 \mathrm{~cm}$.

\section{Clé}

Partie de préhension d'une clé (fer) constituée par une embase à bords convergents surmontée d'un anneau et prolongée par une tige brisée. L. inc. $8 \mathrm{~cm}$.

Ces trois clés sont à rapprocher du type $5 \mathrm{de} \mathrm{H}$. Masurel (1979, p. 236-237, tableau typologique). Ce modèle fonctionne par retrait (Halbout et al., 1987, p. 64 et 71).

056. Clé (Haut-Empire - Niveau de circulation F124/ F125)

Anneau (fer) surmontant une tige rectangulaire coudée en " $\mathrm{U}$ " dont le panneton courbe comporte trois dents tournées vers l'anneau. Ht. 5,6 cm; L. 5,1 cm. Ce modèle semble fonctionner par soulèvement (Halbout et alii 1987, p. 65 et 73$)$.

057. Clé (Haut-Empire - Dépotoir 2)

L'anneau (fer) est percé dans une embase massive à bords parallèles. L'extrémité fonctionnelle se compose de trois dents perpendiculaires à une platine. La platine, corrodée, semble avoir comporté trois pertuis. L. 17,7 cm; $1.4,7 \mathrm{~cm}$; L. dents $4,3 \mathrm{~cm}$. Ce modèle appartient au type $13 \mathrm{Cvi}$ de Masurel (1979, p. 259-261, tableau typologique). Il semble fonctionner par soulèvement (Halbout et al., 1987, p. 65 et 73).

\section{Clé?}

Objet (fer) présentant un emmanchement à douille fermée, brisé dans sa partie supérieure. Il se prolonge par une tige martelée en section quadrangulaire puis en plaque dans l'axe de l'épaisseur. Cette plaque est brisée. L. 10,4 cm; 1. douille $1,2 \mathrm{~cm}$; 1 . plaque $2,5 \mathrm{~cm}$; ép. plaque $0,2 \mathrm{~cm}$. Même si l'usage d'une douille est rare sur une clé, cet exemplaire pourrait correspondre à une clé à rotation.

\section{Clé}

Clé (alliage base cuivre) présentant une embase de section rectangulaire à bords concaves et perforée au niveau de son extrémité supérieure. La tige de section circulaire est perforée. Le panneton se développe à sa perpendiculaire; il dispose d'un rouet, d'un panneton et d'une dent. De petites incisions ornent les bords inférieur et supérieur du panneton. L. 4,2 cm; 1. 2,2 cm; Ht. 2,1 cm; ép. 0,7 cm. Ce type de clé fonctionne par rotation.

Ce système est utilisé durant la période gallo-romaine, d'abord sur des cadenas et, à partir du rv $v^{e}$ siècle, sur le mobilier et l'huisserie (Feugère et al., 1992, p. 30). Les dimensions de 
cette pièce suggèrent une utilisation comme clé de cadenas ou de petit mobilier (coffret?) (ibid.; Masurel 1979, p. 135).

060. Couverture de serrure (Haut-Empire - Niveau de circulation F124/F125)

Cette serrure (fer) se compose d'une plaque de couverture comportant des clous destinés à assurer sa fixation. La plaque est incomplète; néanmoins on y distingue un anneau en " $U$ » (conduit) fixé sur celle-ci. Un fragment de tige plate est situé entre l'anneau et la plaque de couverture (ressort?). Une des extrémités présente l'amorce de deux orifices rectangulaire, disposés parallèlement mais séparés l'une de l'autre. L. inc. $10,4 \mathrm{~cm} ; 1.7,3 \mathrm{~cm}$.

061. Couverture de serrure (non dessinée)

Fragment de plaque de couverture (fer) composé d'une tôle de section rectangulaire, brisée, dont on ne distingue plus qu'une perforation destinée à sa fixation.

\section{Serrure}

Cette serrure (fer) se compose d'une plaque destinée à assurer sa fixation sur la porte (A). Des clous sont situés aux extrémités. Deux orifices rectangulaires y sont aménagés : l'un est disposé perpendiculairement au pêne et dans son prolongement (auberonnière, B); le second lui est parallèle et se situe au dessus (passage de la clé). Ce dernier est découpé dans la plaque sur trois côtés, la plaque étant repliée à la perpendiculaire du quatrième bord afin de former un guide pour le pêne. Le pêne (C) se compose d'une plaque de section rectangulaire prolongée par une tige circulaire à chaque extrémité. Sa translation est assurée par les conduits (D) qui le maintiennent sur la plaque et par les trois gardes destinées à recevoir les dents de la clé. Le ressort à lame est disposé entre le pêne et la plaque. Plaque de couverture : L. $17,8 \mathrm{~cm} ; 1.9,5 \mathrm{~cm}$. Pêne : L. totale $13,9 \mathrm{~cm}$; L. plaque $5,55 \mathrm{~cm} ; 1$. plaque $4,5 \mathrm{~cm}$.

Ce système de serrure fonctionne par retrait (Halbout et al., 1987, p. 64). La perforation perpendiculaire située sur la couverture de serrure peut correspondre à une auberonnière (ibid., p. 66 et 75). Cet aménagement doit être destiné à recevoir l'auberon d'un moraillon, la tige prolongeant le pêne étant destinée à assurer son blocage.

\section{Serrure}

Cette serrure (fer) se compose d'une plaque de couverture présentant une perforation dans chaque coin afin d'assurer sa fixation. La plaque est incomplète, néanmoins on y distingue deux orifices rectangulaires : l'un est disposé perpendiculairement au pêne et dans son prolongement (auberonnière), le second lui est parallèle et se situe au dessus (passage de la clé). Ce dernier est découpé dans la plaque sur trois côtés, la plaque étant repliée à la perpendiculaire du quatrième bord afin de former un guide pour le pêne. Le pêne se compose d'une plaque de section rectangulaire prolongée par une tige circulaire à chaque extrémité. Sa translation est assurée par les conduits qui le maintiennent sur la plaque et par les gardes destinées à recevoir les dents de la clé. Le ressort à lame est disposé entre le pêne et la plaque. Plaque de couverture : L. $17,3 \mathrm{~cm} ; 1.7,9 \mathrm{~cm}$. Pêne : L. totale $20,6 \mathrm{~cm}$; L. plaque $6,6 \mathrm{~cm} ; 1$. plaque $4,2 \mathrm{~cm}$. Ce système de serrure fonctionne par retrait, comme l'exemplaire précédent.

\section{Gâche}

Élément (fer) de section quadrangulaire replié en forme de « $U$ ». Les extrémités sont effilées et tordues. L. 3,8 cm; 1 . 3,2 cm; ép. $0,6 \mathrm{~cm}$. Il s'agit d'un type de crampon probablement utilisé comme gâche (Mitard, 1993, p. 384-385; Halbout et al., 1987, p. 64).

\section{Loquet à ressort}

Élément (fer) constitué d'une tige de section rectangulaire surmontée d'une tête épaisse. Les bords convergent vers une extrémité formée par le repli de la tige sur elle-même, découpée en trois lamelles. L. 9,6 cm; L. lamelles $6,1 \mathrm{~cm}$; 1. 2,9 cm; ép. max. $0,8 \mathrm{~cm}$.

\section{Loquet à ressort}

Plaque (fer) de section quadrangulaire découpée en trois lamelles dans le sens de la longueur. Elle est repliée sur ellemême au niveau d'une extrémité puis est brisée. L. inc. $6,7 \mathrm{~cm} ; 1.2,9 \mathrm{~cm}$; ép. 0,25 cm. Ces éléments appartiennent au mécanisme d'une serrure (Eneau, 2002, p. 41).

\section{Aménagement bydraulique}

067. Fragment de frette (non dessiné)

Ruban (fer) muni d'une arête centrale sur la face externe et présentant un profil légèrement courbe à mi-longueur. Cet élément est incomplet. L. inc. $5,5 \mathrm{~cm} ; 1$. inc. $2,2 \mathrm{~cm}$.

\section{Frette de canalisation (fig. 5)}

Ruban (fer) soudé en cylindre et muni d'une arête centrale sur la face externe. $1.1,5 \mathrm{~cm}$; D. 9 à $9,5 \mathrm{~cm}$.

\section{Frette de canalisation (non dessinée)}

Fragment de ruban (fer) soudé en cylindre et muni d'une arête centrale sur la face externe. $1.2,7 \mathrm{~cm}$; D. $\min .10,6 \mathrm{~cm}$. Les frettes permettent la jonction entre les conduites d'eau en bois (Halbout et al., 1987, P. 55).

\section{Luminaire}

070. Chandelier à broche (fig. 5)

Élément (fer) à douille fermée prolongée par une tige coudée et effilée vers la pointe. L. 9,5 cm; Ht. 6,7 cm; D. max. 


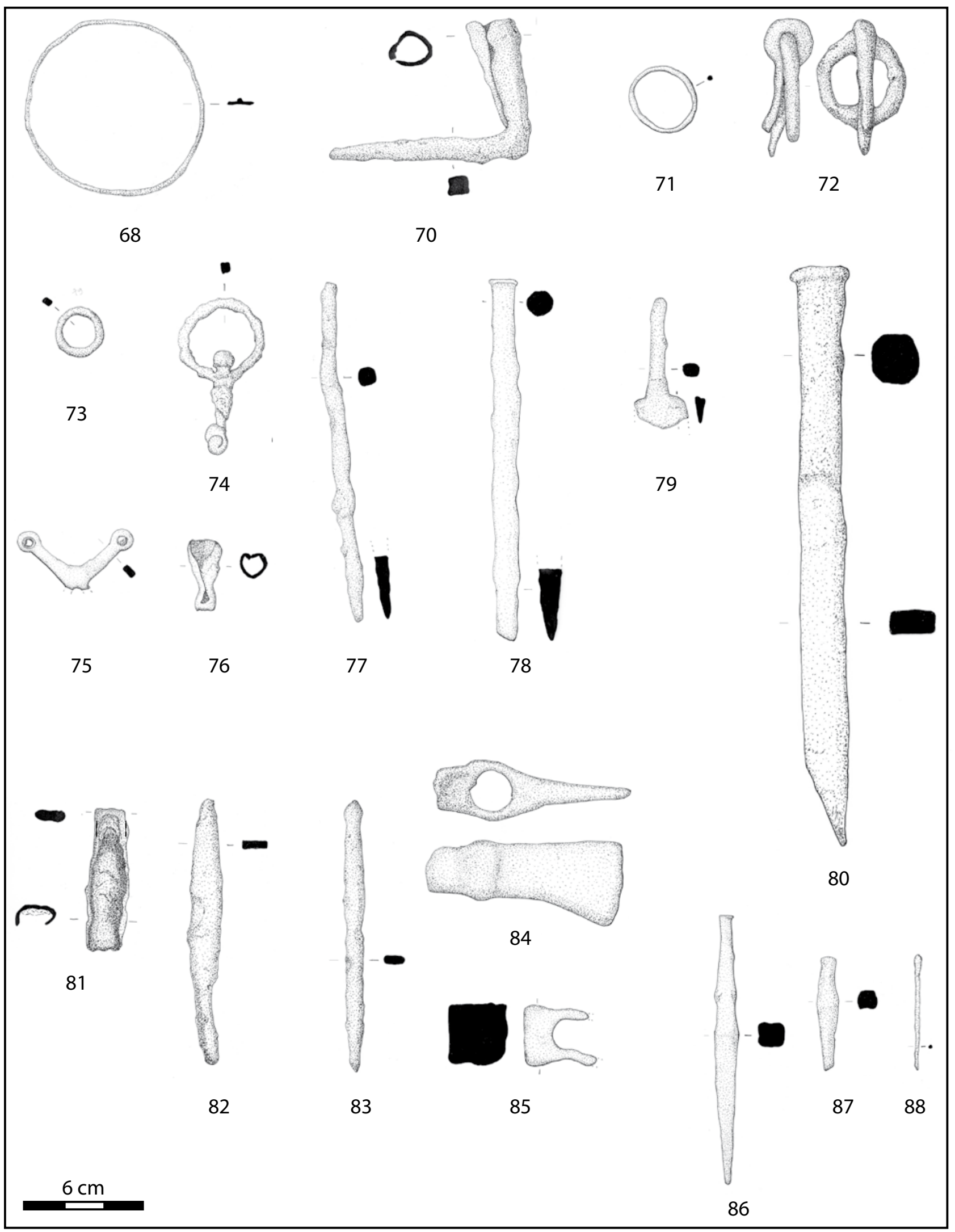

Figure 5 : Élements de système hydraulique (68), de luminaire (70), de suspension ou de liaison (71-75), d'outillage (76-88). Figure 5: Components of hydraulic system (68), of light (70), of suspension or connexion devices (71-75), of tools (76-88). 
douille 2,3 cm; D. int. 1.1 à 1,4 cm. Ce type de chandelier est fixé dans une pièce d'huisserie ou dans la maçonnerie à l'aide de son extrémité appointée (Hofmann 1985, p. 78, pl. 37 n 8-9; Roussel 1979, p. 50, pl. 29 n 202).

\section{Suspensions et liaisons (fig. 5)}

Ces éléments constituent des pièces de liaison fixes ou mobiles pouvant être associé ou fixer sur différents supports (diverses chaînes, anneau fixé à la maçonnerie...).

\section{Anneau}

Anneau (alliage base cuivre) de forme ovale et de section sub-circulaire. Deux parties situées à l'opposé l'une de l'autre présentent une usure importante marquée par une nette diminution de leur section. L. $3,5 \mathrm{~cm} ; 1.3,2 \mathrm{~cm}$; l. et ép. tige $0,3 \mathrm{~cm}$. L'usure de cet anneau suggère une utilisation comme élément de jonction (anneau de chaîne?).

072. Anneau dit d'écurie ou d'amarrage

Anneau (fer) de section circulaire avec patte-fiche de scellement. La fiche est formée par le repli symétrique d'une tige de section carrée, appointée à chaque extrémité. D. ext. anneau 4,6 cm; ép. 0,9 cm; L. fiche $6,55 \mathrm{~cm}$. Ce type d'anneau sert à attacher les animaux et à amarrer les bateaux (Guillaumet, 2003, p. 129). Néanmoins, leur usage a pu être varié : anneau de chaîne... (Halbout et al., 1987, p. 118).

\section{Anneau}

Anneau (fer) de forme circulaire et de section rectangulaire. D. ext. $2 \mathrm{~cm}$; D. int. $7 \mathrm{~cm}$; ép. $0,3 \mathrm{~cm}$.

\section{Tourillon}

Anneau (fer) de section sub-carrée, martelé et percé pour permettre le passage d'un émerillon. Ce dernier se présente sous la forme d'une tige à tête pleine et bombée d'un côté. La seconde extrémité semble être repliée en boucle; un fragment de tige brisée courbée en « $U$ » s'y insère. D. 4,2 cm; L. émerillon $5,3 \mathrm{~cm}$. La forme de la tête de l'émerillon lui permet de tourner librement sur lui-même (Eneau, 2002, p. 20).

De tels éléments constituent une partie d'un assemblage. Ils sont utilisés comme extrémité de chaîne et parfois pour suspendre des récipients au-dessus du feu (Manning, 1985, p. 138-139). Ces pièces évitent la torsion des chaînes (Guillaumet 2003, p. 141).

075. Élément de suspension (de balance portative?)

Élément (alliage base cuivre) en forme de « $\mathrm{V}$ » présentant un anneau méplat à chacune des trois extrémités. Un des anneaux est fragmenté, un autre présente des traces de fer corrodé. Enfin, leur perforation est biconique et l'un d'entre eux présente une incision : usure ou fonction de blocage? Le pourtour extérieur des anneaux est incisé; leur fonction a pu être ornementale et usuelle. L. inc. branche $4,3 \mathrm{~cm}$; Espace extérieur $5,7 \mathrm{~cm}$; ép. $0,3 \mathrm{~cm}$.

Un objet comparable, présentant trois anneaux répartis à chaque angle d'un triangle isocèle, a été découvert à Frénouville (Calvados : Pilet 1987, p. 9); il était associé à un crochet et à trois poids en alliage cuivreux, ce qui a permis de déterminer l'ensemble comme une petite balance portative. Le présent exemplaire pourrait avoir eu un usage similaire.

\section{Outillage}

L’outillage représenté ici renvoie vraisemblablement au travail de différentes matières mais plus particulièrement à celui du bois. Les couteaux, d'un usage quotidien, peuvent avoir eu des usages domestiques comme la cuisine, mais leur rôle ne se restreint pas à ce domaine.

\section{Outillage artisanal (fig. 5 et 6)}

\section{Petit ciseau?}

Outil (fer) à douille fermée à la base puis martelée en spatule. Les deux extrémités sont tronquées. L. inc. $3,4 \mathrm{~cm}$; D. ext. douille $1,8 \mathrm{~cm} ; 1$. inc. spatule $1,5 \mathrm{~cm}$. Il s'agit d'un petit outil à douille : petit ciseau?

\section{Ciseau?}

Tige (fer) de section rectangulaire présentant un léger décalage de son axe sur le dernier tiers de sa longueur. Cette partie est très attaquée par la corrosion; elle est aplatie et légèrement effilée, mais de manière moins marquée que la seconde extrémité. L. $17 \mathrm{~cm} ; 1.0,9 \mathrm{~cm}$; ép. $0,95 \mathrm{~cm}$. L'extrémité la plus fine suggère une utilisation comme outil de type ciseau (Manning 1985, pl. 6).

078. Ciseau (Haut-Empire - Niveau de circulation F124/ F125)

Outil (fer) présentant une tête et une tige de section circulaire. La tige est martelée en section rectangulaire et biseautée sur l'extrémité utile. L. 18,5 cm; D. max. $1,5 \mathrm{~cm}$. Il s'agit d'un ciseau utilisé pour le travail des métaux ou du bois (Manning 1985, p. 9).

079. Outil à soie (ciseau ou grattoir?)

Tige (fer) de section rectangulaire, évasée et achevée en biseau sur une extrémité brisée. L. inc. $6,5 \mathrm{~cm} ; 1.2,5 \mathrm{~cm}$; ép. 0,6 à $0,2 \mathrm{~cm}$. Outil à soie de type ciseau ou grattoir. 


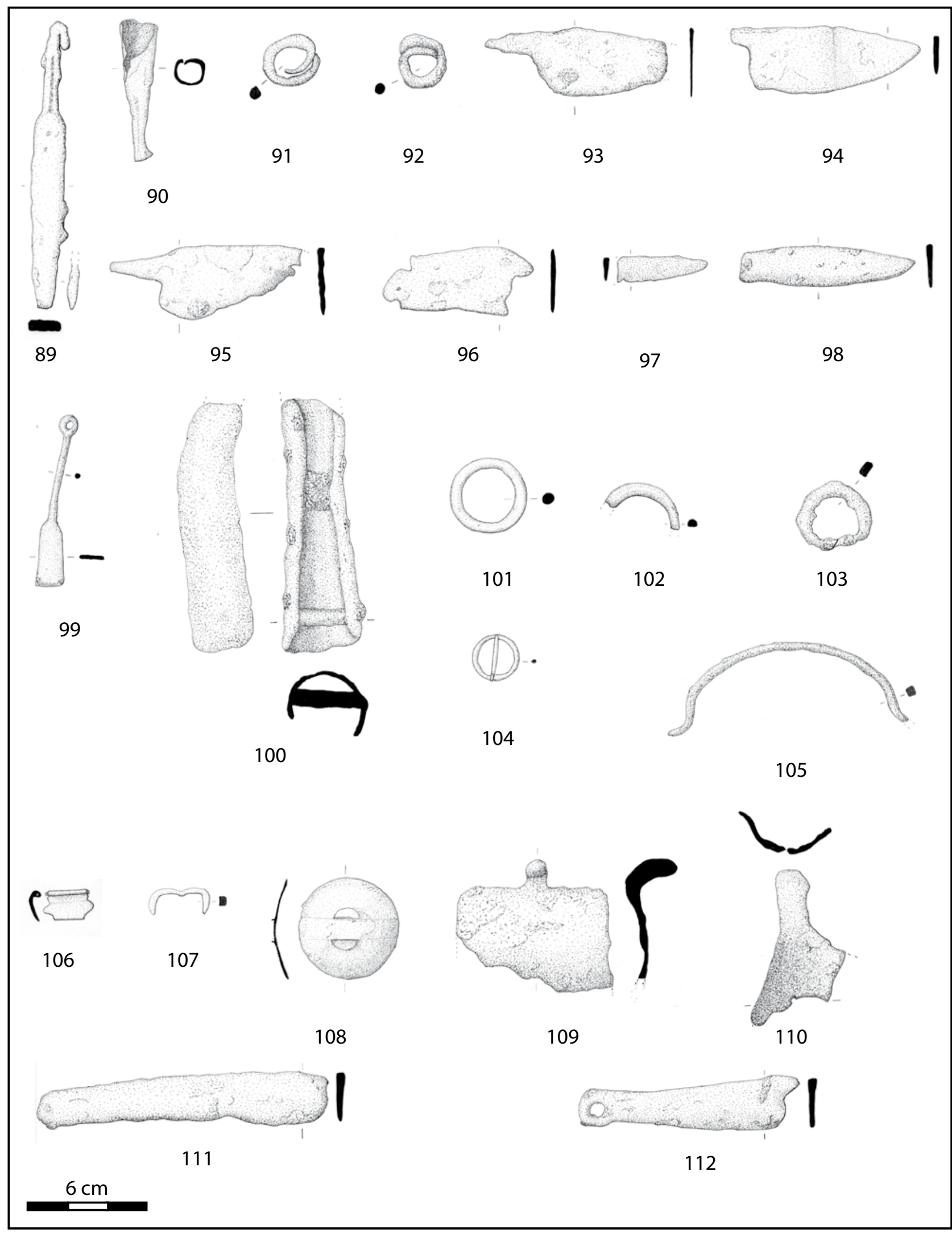

Figure 6 : Éléments d'outillage, suite (89-92), d'équipement domestique (93-98), inclassables et indéterminés (101-112). Figure 6: Further components of tools (89-92); of domestic materials (93-98); unclassificable and undetermined (101-112). 
080. Bédane (Haut-Empire - Niveau de circulation F124/

F125)

Élément (fer) dont la partie de frappe et de préhension présente une forme octogonale. La tige est ensuite martelée en section rectangulaire et le tranchant est pris dans l'épaisseur de la tige. L. $29,5 \mathrm{~cm} ; 1.3,2 \mathrm{~cm}$. Cet outil est utilisé pour le travail du bois, le tranchant en un seul biseau permet d'obtenir des parois verticales lors du creusement de mortaises (Duvauchelle 1990, p. 22-23; Manning 1985, p. 20-24, fig. $\left.4 \mathrm{n}^{\circ} 5\right)$.

\section{Fragment de gouge?}

Fragment (fer) de tôle de section rectangulaire repliée sur chaque bord, prenant ainsi une forme en " $U$ ». Sa profondeur et sa largeur diminuent vers une extrémité. Le profil est courbe, particulièrement au niveau de l'extrémité la moins large. L. inc. $7,1 \mathrm{~cm} ; 1.2 \mathrm{~cm}$; ép. $0,8 \mathrm{~cm}$. Il s'agit vraisemblablement de la partie utile d'une gouge (Manning, 1985, p. 24-25).

082. Lime (Haut-Empire - Niveau de circulation F124/ F125)

Outil (fer) de forme lancéolée à emmanchement à soie. Il est tordu et altéré par la corrosion; néanmoins sa surface présente ponctuellement quelques stries. L. $13,1 \mathrm{~cm}$; $1.1,1 \mathrm{~cm}$; ép. $0,4 \mathrm{~cm}$. Il s'agit vraisemblablement d'une petite lime (Duvauchelle, 1990, p. 12). Les lames des limes romaines sont généralement comprises entre 10 et $20 \mathrm{~cm}$.

\section{Lime}

Tige (fer) de section rectangulaire effilée vers chaque extrémité. Quelques stries sont ponctuellement visibles sur sa surface. Une des extrémités est tronquée. L. inc. $13,5 \mathrm{~cm}$; $1.1,1 \mathrm{~cm}$; ép. 0,4 à $0,3 \mathrm{~cm}$. Il s'agit d'une petite lime (Manning, 1985, p. 11, A37-38).

084. Hache (Haut-Empire - Niveau de circulation F124/ F125)

Hache (fer) à talon. Le dos est légèrement convexe et le fil est concave. L'œil d'emmanchement, circulaire, présente un léger décrochement avec le talon, qui est brisé sur une face. L. $9,8 \mathrm{~cm}$; Ht. tranchant $4,2 \mathrm{~cm} ; 1 . \max .3,1 \mathrm{~cm}$. Cette hache se rapproche du type 3 de W. H. Manning (1985, p. 14-16) dans sa forme générale; néanmoins, le décrochement formé par deux petites œillères situées sous l'œil pourrait la rapprocher davantage du type $2 \mathrm{~B}$ des haches d'Avenches (Duvauchelle, 1990, p. 15-16). Toutefois ce dernier modèle, daté de l'Antiquité tardive, se caractérise par des œillères triangulaires et l'état de fragmentation de notre exemplaire ne permet pas de déterminer leur forme originelle.

\section{Talon de hache ou de marteau?}

Talon (fer) et amorce d'œil d'emmanchement brisé, dont chaque bord diverge légèrement. L. inc. $3,7 \mathrm{~cm} ; 1.2,8 \mathrm{~cm}$; Ht. 2,6 cm.

\section{Poinçon}

Octaèdre (fer) formé de deux pyramides opposées à la base. Une des extrémités est effilée et la seconde présente un bourrelet et une face plate, caractéristiques d'une utilisation comme surface de frappe. L. $13,4 \mathrm{~cm} ; 1.1,3 \mathrm{~cm}$; ép. $1,3 \mathrm{~cm}$. La morphologie générale et la présence d'une surface de frappe suggèrent une utilisation comme poinçon. La forme de son manche semble couramment utilisée pour ce type d'outil (Duvauchelle 1990, p. 86, n²1-23).

\section{Alêne?}

Tige (fer) de section carrée, effilée et appointée vers une extrémité et brisée vers la seconde, elle-même légèrement effilée. L. inc. $5,7 \mathrm{~cm} ; 1.1,1 \mathrm{~cm}$; ép. $1 \mathrm{~cm}$. La forme de l'objet est trop irrégulière pour pouvoir déterminer clairement sa fonction. Néanmoins sa morphologie rappelle celle de certains poinçons utilisés comme alêne (Duvauchelle, p. 3839 , type $3 b, n^{\circ} 159,160,162$ ).

\section{Aiguille?}

Tige (fer) de section circulaire effilée vers une extrémité. Les deux extrémités sont brisées. L. inc. $5,6 \mathrm{~cm} ; \mathrm{D} .0,4 \mathrm{~cm}$. Il s'agit vraisemblablement d'une aiguille mais, en l'absence de l'extrémité comportant le chas, rien ne permet de l'affirmer.

\section{Dent de râteau?}

Élément (fer) de section rectangulaire, légèrement effilé vers l'extrémité inférieure et martelé en soie dans sa partie supérieure. La soie forme un épaulement avec la tige et s'effile vers sa seconde extrémité repliée sur elle-même. L. $14,1 \mathrm{~cm} ; 1.1,6 \mathrm{~cm}$; ép. $0,35 \mathrm{~cm}$. La soie courbée et l'épaulement contribuent à assurer sa fixation. La morphologie générale peut suggérer une utilisation comme dent de râteau (Manning, 1985, p. 59, n F64 et 66).

090. Talon? (Haut-Empire - Niveau de circulation F124/ F125)

Élément (fer) dont la partie utile se présente sous la forme d'une tige effilée de section quadrangulaire. Sa partie supérieure, destinée à l'emmanchement, est martelée en douille. Ht. $7 \mathrm{~cm} ; 1.1,9 \mathrm{~cm}$; ép. douille $0,2 \mathrm{~cm}$. Ce type d'objet est couramment interprété comme talon destiné entre autres à protéger l'extrémité du manche en bois d'outils ou de lances (Manning, 1985, p. 140-141, pl. 66). 


\section{Virole à spirale}

Tige (fer) de section circulaire légèrement effilée aux extrémités et enroulée en spirale. D. $0,5 \mathrm{~cm}$; Ht. $2 \mathrm{~cm}$.

\section{Virole à spirale}

Tige (fer) de section circulaire légèrement effilée aux extrémités et enroulée en spirale. D. $0,5 \mathrm{~cm}$.

Les viroles à spirales servent à enserrer l'extrémité d'un manche (Eneau, 2002, p. 29; Manning, 1985, pl. 67).

\section{Outillage domestique (fig. 6)}

\section{Couteau}

Couteau (fer) présentant un emmanchement à soie situé dans le prolongement du dos. Le dos, le talon et le fil sont légèrement convexes. Le dos et le fil convergent vers la pointe qui est brisée. L. inc. $8,9 \mathrm{~cm} ; 1.3,3 \mathrm{~cm}$; ép. $0,3 \mathrm{~cm}$.

\section{Couteau}

Couteau (fer) présentant un emmanchement à soie situé dans le prolongement du dos. Le dos et le fil sont légèrement convexes et convergent vers la pointe. Le talon marque un angle droit avec la soie brisée. La lame est tordue. L. inc. $9,4 \mathrm{~cm} ; 1.3,2 \mathrm{~cm}$; ép. $0,4 \mathrm{~cm}$.

Cette forme de couteau se caractérise principalement par la largeur de sa lame ainsi que par la convexité du dos et surtout du fil qui remonte vers la pointe. Ces deux couteaux pourraient appartenir à une variante de faible dimensions $\mathrm{du}$ type 12 de W. H. Manning (1985, p. 108, 114, 115).

\section{Couteau}

Couteau (fer) présentant un emmanchement à soie situé dans le prolongement du dos. Le dos et le fil sont légèrement convexes et convergent vers la pointe, brisée. Le talon marque un angle droit avec la soie. L'angle qu'il forme avec le fil est arrondi. La lame triangulaire est tordue et brisée. L. inc. $9,4 \mathrm{~cm} ; 1.3,6 \mathrm{~cm}$; ép. $0,4 \mathrm{~cm}$. Cette forme de couteau se caractérise principalement par la largeur de sa lame et le fil qui remonte vers la pointe. Son état de conservation rend difficile toute classification.

\section{Couteau pliant}

Couteau (fer) présentant un emmanchement perforé pour permettre le passage d'une goupille. Le dos marque un angle droit avec l'emmanchement. Son profil est droit puis se courbe vers la pointe. Le fil est usé et la pointe est brisée. L. inc. 7,4 cm;1. 3,3 cm; ép. 0,2 cm. Il s’agit d'un modèle de canif pliant dont la lame pivote pour se replier vers le manche (Roussel, 1979, pl. 30).

\section{Lame}

Lame (fer) de forme et de section triangulaires. Le fil est légèrement convexe et le dos converge vers la pointe. La lame est légèrement tordue et l'extrémité la plus large est brisée. L. inc. 4,6 cm; $1.1,2 \mathrm{~cm}$; ép. 0,3 à $0,1 \mathrm{~cm}$.

\section{Lame de couteau}

Lame (fer) de forme lancéolée et de section triangulaire. Le dos et le fil ont un profil convexe. La soie est brisée. L. inc. $8,6 \mathrm{~cm} ; 1.2 \mathrm{~cm}$; ép. $0,4 \mathrm{~cm}$.

\section{Outils indéterminés (fig. 6)}

\section{Outil}

Élément (alliage base cuivre) présentant une tige de section sub-circulaire prolongée d'un côté par un anneau méplat perforé et de l'autre par une spatule. La spatule présente une section rectangulaire et une forme trapézoïdale. Sa particularité réside dans son extrémité courbée dont chaque bord est orné d'une griffe recourbée vers la tige. Cet objet est tordu. L. $8,8 \mathrm{~cm} ; 1.1,3 \mathrm{~cm}$; ép. $0,35 \mathrm{~cm}$. En l'absence d'éléments de comparaison, il est difficile d'attribuer une fonction à ce petit outil : accessoire de médecine, utilisation pour des travaux manuels particuliers...

100. Outil à emmanchement à douille ouverte (HautEmpire - Niveau de circulation F124/F125)

Plaque (fer) martelée en douille ouverte de forme légèrement conique. L'extrémité destinée à assurer l'emmanchement est traversée par un clou. La seconde extrémité se courbe juste avant la cassure. L. inc. 12,4 cm; D. max. $4 \mathrm{~cm}$. Cet objet massif présente une forte résistance mécanique. S'agit-il d'un outil agricole de type soc d'araire?

\section{Inclassables et indéterminés}

\section{Objets entiers (fig. 6)}

En l'absence des éléments auxquels ils étaient associés l'usage précis de certains objets ne peut être défini.

101. Anneau (Bas-Empire - Dépotoir DP2)

Anneau (alliage base cuivre) de forme et de section circulaires. D. ext. 3,8 cm; 1. 0,6 cm; ép. moy. 0,6 cm.

\section{Anneau (Bas-Empire - Dépotoir DP2)}

Anneau (alliage base cuivre) de forme probablement circulaire et de section ovale. Seule une moitié est conservée. Une des faces présente des stries dues à l'usure et une partie de l'autre face présente une surface à trois pans atténués. D. ext. $3,9 \mathrm{~cm} ; 1.0,6-0,7 \mathrm{~cm}$; ép. moy. $0,4 \mathrm{~cm}$.

De tels anneaux sont d'un usage varié : maillons de chaîne, pour le maintien de bijoux, comme élément de parure, pour 
le harnachement... (Crummy, 1983, p. 161). Il est difficile de leur attribuer une fonction précise.

\section{Anneau}

Anneau (fer) de forme circulaire et de section rectangulaire. $1.3,7 \mathrm{~cm}$; Ht. inc. $3,3 \mathrm{~cm}$. La morphologie de cet anneau suggère une utilisation comme boucle ou comme élément de préhension.

\section{Boucle (Bas-Empire - Dépotoir DP2)}

Anneau (alliage base cuivre) de forme circulaire et de section triangulaire, présentant une petite portion plus fine permettant à un ardillon de venir s'y fixer et de pivoter. D. ext. $2,3 \mathrm{~cm} ; 1.0,25 \mathrm{~cm}$; ép. $0,2 \mathrm{~cm}$; L. ardillon $2,45 \mathrm{~cm}$. Cette boucle peut avoir été utilisée pour relier des courroies de cuir, de passementerie ou d'étoffe sur des accessoires du vêtement ou de harnachement (Halbout et al., 1987, p. 182).

\section{Poignée}

Tige (fer) de section carrée, courbée et martelée à chaque extrémité pour amorcer un crochet. Les extrémités sont brisées. L. inc. $11,8 \mathrm{~cm}$; Ht. 4,5 cm; l. et ép. 0,4 cm.

\section{Objets fragmentés (fig. 6 et 7 )}

\section{6. Élément de charnière?}

Tôle (alliage base cuivre) de section rectangulaire repliée sur elle-même pour former une charnière dans laquelle s'insère une goupille sur une extrémité. Elle se développe de l'autre côté en une tôle courbe présentant deux protubérances latérales. L. $2,4 \mathrm{~cm}$; Ht. 1,55 cm; ép. charnière $0,45 \mathrm{~cm}$.

\section{Objet indéterminé}

Élément (alliage base cuivre) en forme de " $\mathrm{M}$ » dont chaque extrémité est légèrement biseautée. L. $3 \mathrm{~cm}$; Ht. $1,3 \mathrm{~cm}$; ép. $0,4 \mathrm{~cm}$. Anneau en « 8 » coupé en deux?

\section{Ornement}

Élément (fer) de section rectangulaire dont la forme est circulaire et le profil convexe. La face externe présente un revêtement en alliage jaune (laiton?) conservé sur sa partie sommitale. Ce revêtement, disposé symétriquement de part et d'autre d'une bande non recouverte, se développe à la perpendiculaire de la tôle le long de cette bande. D. $5 \mathrm{~cm}$; ép. $0,1 \mathrm{~cm}$. Toute la surface a pu être initialement recouverte de laiton. La fixation s'effectue sur la face externe (bombée) à l'aide du laiton qui formait probablement un passant.

\section{Objet indéterminé}

Tôle (fer) de section rectangulaire et de profil légèrement courbe. Une des extrémités est brisée et la seconde est prolongée par un crochet. L. inc. 7,1 cm; 1. 7,6 cm; ép. $0,4 \mathrm{~cm}$.

\section{Objet indéterminé}

Élément (fer) composé d'une tige de forme et de section rectangulaires présentant une extrémité arrondie. Elle s'évase et prend un profil en " $\mathrm{V}$ » au niveau de la seconde extrémité, brisée. L. inc. 7,4 cm; 1. 5,7 cm ; ép. 0,4-0,2 cm.

111. Objet indéterminé (Haut-Empire - Niveau de circulation F124/F125)

Tige (fer) de section triangulaire dont la largeur décroît vers une extrémité. Son profil est légèrement courbe. L. $14,3 \mathrm{~cm} ; 1.2,3-1,7 \mathrm{~cm}$; ép. 0,4-0,1 cm.

112. Objet indéterminé (Haut-Empire - Niveau de circulation F124/F125)

Élément (fer) de section et de forme sub-triangulaire, achevé d'un côté par un anneau repercé dans la tige et de l'autre par une petite soie située dans le prolongement du bord le plus épais. La surface originelle est altérée par la corrosion. L. 10,7 cm; 1. 2,5 cm; ép. 0,5 cm. La section triangulaire ne semble pas s'expliquer par une volonté d'utilisation comme outil tranchant car le bord le plus mince atteint $0,2 \mathrm{~cm}$. La morphologie ne semble pas correspondre à un outil.

\section{Indéterminé}

Plaque (fer) de section rectangulaire à bords divergents. Une des faces comporte un sillon longitudinal en son milieu. Il ne se poursuit pas dans la partie supérieure, plus large, mais se prolonge jusqu’à la seconde extrémité à partir de laquelle la plaque est martelée en deux branches plus fines. Les extrémités sont tronquées. L. inc. $5,5 \mathrm{~cm} ; 1.3,9 \mathrm{~cm}$; ép. $0,6 \mathrm{~cm}$.

114. Indéterminé (Haut-Empire - Niveau de circulation F124/F125)

Tige (fer) de section rectangulaire courbée à $90^{\circ}$ d'un côté et prolongé par un emmanchement à douille de l'autre. Aucune extrémité n'est conservée. L. inc. $7,1 \mathrm{~cm} ; 1$. douille $1,7 \mathrm{~cm}$.

\section{Indéterminé}

Élément (fer) présentant une tige de section ovale surmontée d'un anneau d'un côté et martelée en section triangulaire plus fine de l'autre. L. $9 \mathrm{~cm} ; 1$. anneau $2 \mathrm{~cm} ; 1.1,1 \mathrm{~cm}$; ép. $0,85 \mathrm{~cm}$.

\section{Indéterminé}

Tige (fer) de section rectangulaire effilée vers une extrémité et dont le profil est courbe. L. inc. $9,5 \mathrm{~cm} ; 1.1,1 \mathrm{~cm}$; ép. $0,4 \mathrm{~cm}$. 


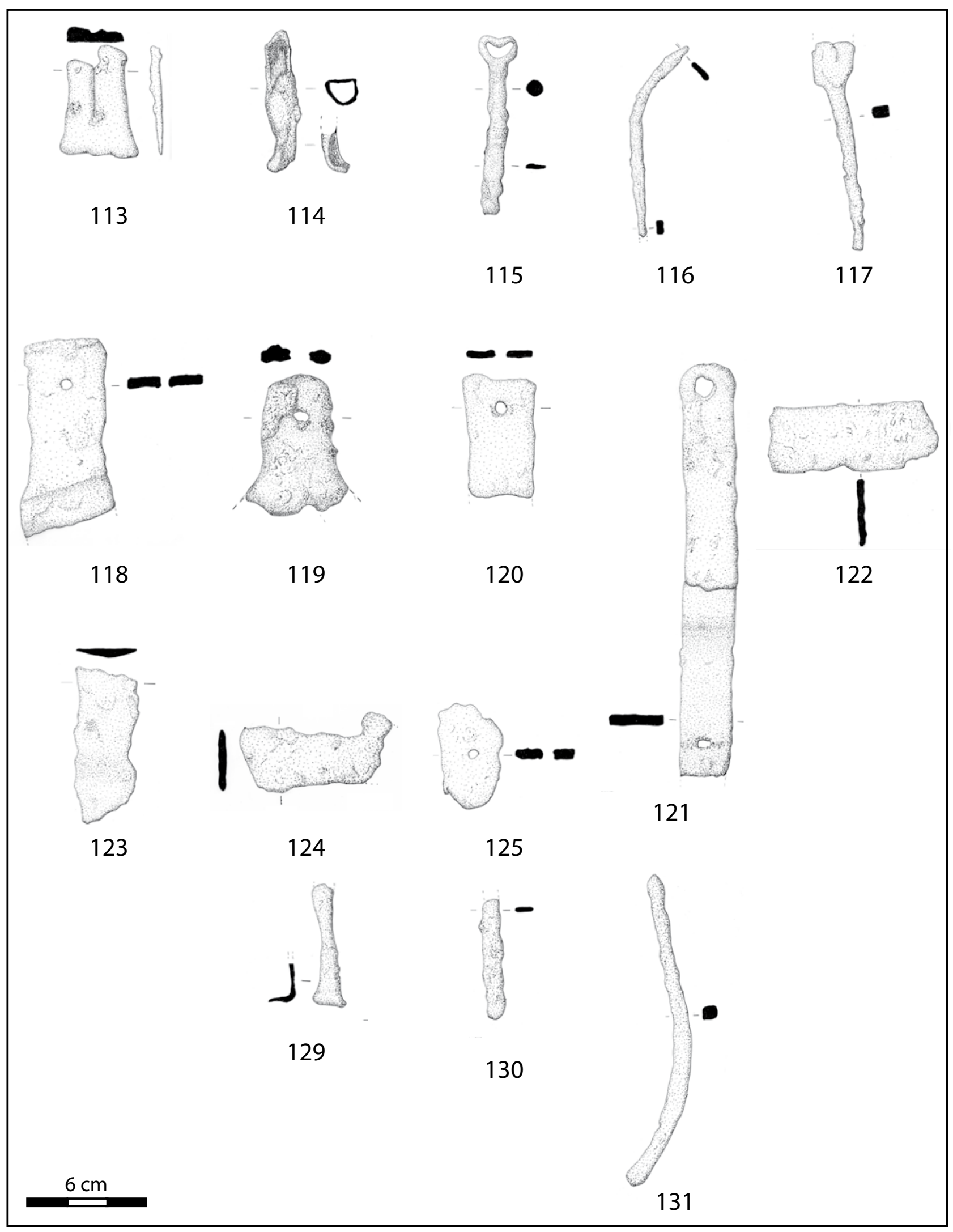

Figure 7 : Inclassables et indéterminés, suite (113-125 et 129-131).

Figure 7: Unclassificable and undetermined, continued (113-125 \& 129-131). 


\section{Indéterminé}

Tige (fer) de section rectangulaire, martelée en plaque au niveau d'une extrémité et effilée vers la seconde. La partie utile de cet objet est représentée par la plaque qui est brisée. L. inc. $10,6 \mathrm{~cm}$; l. plaque $2,2 \mathrm{~cm}$; ép. plaque $0,3 \mathrm{~cm}$.

\section{Fragment d'objet}

Plaque (fer) de section rectangulaire dont les bords s'évasent vers son extrémité brisée. Elle forme un léger décrochement avec cette partie brisée dont la section est moins épaisse et dont les bords s'évasent. L'autre extrémité présente une perforation circulaire. L. inc. $9,6 \mathrm{~cm} ; 1.4,7 \mathrm{~cm}$; ép. $0,65-0,3 \mathrm{~cm}$. Il s'agit de la partie de préhension ou de fixation d'un objet dont la fonction reste indéterminée.

\section{Fragment d'objet}

Élément (fer) composé d'une plaque de section rectangulaire à bords divergents. Il est percé d'un orifice irrégulier au niveau d'une extrémité et la seconde est brisée. L. inc. $7 \mathrm{~cm}$; 1. $5,2 \mathrm{~cm}$; ép. $0,4 \mathrm{~cm}$. Il s'agit de la partie de préhension ou de fixation d'un objet dont la fonction reste indéterminée.

\section{Ferrure?}

Tige (fer) de section rectangulaire dont les bords convergent légèrement vers une extrémité comportant une perforation. Aucune extrémité n'est conservée. L. inc. $6,1 \mathrm{~cm}$; $1.3,7 \mathrm{~cm}$; ép. $0,25 \mathrm{~cm}$.

121. Ferrure? (Haut-Empire - Niveau de circulation F124/ F125)

Tige (fer) de section et de forme rectangulaire perforée au niveau d'une extrémité. Elle présente un profil en " $\mathrm{L}$ " et une autre perforation au niveau de la seconde courbure. Elle s'effile légèrement vers la seconde extrémité qui est brisée. La surface originelle est altérée par la corrosion. L. inc. $26,5 \mathrm{~cm} ; 1.2,6-2 \mathrm{~cm}$; ép. $0,3-0,5 \mathrm{~cm}$. Cet élément a pu constituer la partie de préhension d'un objet ou la partie de fixation d'un élément d'assemblage.

\section{Tôle}

Plaque (fer) de section rectangulaire dont les extrémités sont brisées. L. 8,6 cm; 1. 3,4 cm; ép. 0,2-0,1 cm.

\section{Tôle}

Plaque (fer) de section sub-ovale dont aucun bord n'est conservé. L. inc. 7,9 cm; 1 . inc. $3,1 \mathrm{~cm}$; ép. 0,5-0,4 cm.

\section{Tôle}

Plaque (fer) de section rectangulaire dont les bords sont brisés. Ep. $0,3 \mathrm{~cm}$.

\section{Tôle}

Plaque (fer) de section rectangulaire comportant une perforation circulaire. Aucun bord n'est conservé. Ep. 0,2$0,1 \mathrm{~cm}$.

126. Tôle (non dessinée)

Plaque (fer) de section rectangulaire dont aucun bord n'est conservé. Ep. 0,5 cm.

127. Tôles (non dessinées)

Il s'agit de deux tôles (alliage cuivreux) ne recollant pas.

128. Tôle (non dessinée)

Plaque (alliage cuivreux) rivetée dont aucun bord n'est conservé. Elle est tordue sur elle-même. Ep. 0,05 cm.

\section{Tige}

Tige (fer) de section rectangulaire évasée vers chaque extrémité. L'une d'entre elles est coudée. Les deux extrémités sont brisées. L. inc. $5,9 \mathrm{~cm} ; 1.1,7 \mathrm{~cm}$; ép. 0,3 cm.

\section{Tige}

Tige (fer) de section rectangulaire brisée à chaque extrémité. L. inc. $5,9 \mathrm{~cm} ; 1.1,1 \mathrm{~cm}$; ép. $0,3 \mathrm{~cm}$.

\section{Tige}

Tige (fer) de section rectangulaire, tordue et brisée au niveau d'une extrémité. La seconde extrémité est appointée. L. inc. $15 \mathrm{~cm} ; 1.0,8 \mathrm{~cm}$; ép. $0,7 \mathrm{~cm}$.

132. Tige (non dessinée)

Tige (fer) de section rectangulaire, coudée au niveau d'une extrémité. Les deux extrémités sont brisées. L. inc. 6,9 cm; $1.1,1 \mathrm{~cm}$; ép. $1,1 \mathrm{~cm}$.

\section{Tige (non dessinée)}

Tige (plomb) de section et de forme sub-rectangulaires, brisée et présentant trois incisions médianes. L. inc. $3,1 \mathrm{~cm}$; $1.1,2 \mathrm{~cm}$; ép. $0,7 \mathrm{~cm}$.

\section{Bibliographie}

Bishop, M.-C., 1992 - The early imperial "apron", Journal of Roman Equipment Studies, 3, p. 81-104.

Collectif, 1983 - La civilisation romaine de la Moselle à la Sarre. Vestiges romains en Lorraine, au Luxembourg, dans la région de Trèves et en Sarre, catalogue d'exposition, Paris, Musée du Luxembourg. 
Crummy, N., 1983 - Colchester archaeological report 2 : The Roman small finds from excavations in Colchester 1971-1979, Colchester Archaeological Trust Ltd., 183 p.

Dollfus, M.-A., 1973 - Catalogue des fibules de bronze de HauteNormandie, Paris, Mémoires de l'Académie des Inscriptions et Belles-Lettres (16), 258 p., 57 pl.

Duvauchelle, A., 1990 - Les outils en fer du Musée Romain d'Avenches, Bulletin de l'Association Pro Aventico, 32, 118 p.

Egan, G. and Pritchard, F. (eds.), 1991 - Mediaeval finds from excavations in London: 3. Dress accessories c.1150-c. 1450, London, Museum of London, $410 \mathrm{p}$.

ENEAU, Th., 2002 - Les objets en fer du sanctuaire gallo-romain du Gué-de-Sciaux (Antigny, Vienne), Antigny, Chauvigny, Association des Publications chauvinoises (Mémoire XXI), $114 \mathrm{p}$.

Fauduet, I., 1992 - Musées d'Évreux. Bronzes gallo-romains, Argenton-sur-Creuse, éd. Instrumentum.

Feugère, M., 1985 - Les fibules en Gaule méridionale de la Conquête à la fin du v viècle apr. J.-C., Revue archéologique de Narbonnaise, suppl. 12, 509 p.

Feugère, M., Thauré, M., Vienne, G. et al., 1992 - Les objets en fer dans les collections du musée archéologique de Saintes $\left(I^{r}-X V^{e}\right.$ siècles), Saintes, Musées de Saintes, 113 p.

Feugère, M. et Lambert, P.-Y., 2004 - L'écriture dans la société gallo-romaine, Gallia, 61, p. 1-192.

Guillaumet, J.-P. et Nilesse, O., 2000 - Les petits objets de quelques fermes gauloises : approche méthodologique, in Marion et Blancquaert, G., Les installations agricoles de l'Âge du Fer en France septentrionale, Paris, ENS, Études d'Histoire et d'Archéologie, 6, p. 251-276.

Guillaumet, J.-P., 2003 - Paléométallurgie métallique, méthode d'étude, Dijon/Quétigny, Folio Éditions, 156 p., 75 ill.

Halbout, P., Pilet, Ch. et Vaudour, C., 1987 - Corpus des objets domestiques et des armes en fer de Normandie, du $I^{e r}$ au XV siècles, Caen, Centre archéologique de Normandie (Cahiers des Annales de Normandie, 20), 255 p.

Hofmann, B., 1985 - Inventaire de quincaillerie antique, Guiryen-Vexin, Centre de Recherches archéologiques du Vexin français (Cahier archéologique $\mathrm{n}^{\circ}$ 5), $100 \mathrm{p}$.

KaZANSKI, M., 1996 - Les barbares en Gaule du sud-ouest durant la première moitié $\mathrm{du} \mathrm{v}^{\mathrm{e}}$ siècle, L'occident romain et l'Europe centrale au début de l'époque des Grandes migrations (Colloque international), Caen, université de Caen, p. 15-23.

Koller, A. et Herzog, R., 1998 - Die Alamannen, Stuttgart, éd. Theiss, $129 \mathrm{p}$.

LeClerc, G., 2002 - Fouille programmée annuelle. Sées (Orne) Le Grand-Herbage. Habitat rural gallo-romain, (DFS de fouille programmée), Caen, SRA de Basse-Normandie, 112 p., 33 fig.

Manning, W. H., 1985 - Catalogue of Romano-British Iron Tools, Fittings and Weapons in the British Museum, London, 197 p., $110 \mathrm{pl}$.
Masurel, H., 1979 - Contribution à l'étude de la serrurerie galloromaine, (Mémoire de l'École du Louvre), Paris, 4 tomes, $213 \mathrm{p}$.

MitaRD, P.-H., 1993 - Le sanctuaire gallo-romain de Genainville (Val d'Oise), Guiry-en-Vexin, Éd. du Centre de Recherches archéologiques du Vexin français, p. 384-388.

Oldenstein, J., 1976 - Zur Ausrüstung römischer Auxiliareinheiten, Berichte der Römisch-Germanischen Kommission, 57, p. 49-366.

Paillard, D., 1994 - Les éléments du IV siècle, la nécropole Michelet. Lisieux avant l'An Mil. Essai de reconstitution, catalogue d'exposition, Lisieux, Musée de la ville de Lisieux, 136 p.

Paillard, D. et Alduc-Le Bagousse, A, 1996 - Présence militaire et influences germaniques en Normandie à la fin du $\mathrm{IV}^{\mathrm{e}}$ siècle; les témoins familiaux à Lisieux (Calvados, France), L'occident romain et l'Europe centrale au début de l'époque des Grandes migrations (Colloque international), Caen, université de Caen, p. 25-31.

Philippe, J., 1997 - Les fibules du Bas-Empire de Seine-et-Marne, in Ouzoulias, P. et Van Ossel, P. (dir.), PCR L'époque tardive en Ille-de-France, Document de travail $n^{\circ} 4$, Paris, Diocesis Galliarum, p. 7-21.

Philippe, J., 2000 - Les fibules de Seine-et-Marne du er $^{\text {r }}$ siècle av. J.-C. au ve siècle apr. J.-C., Nemours, Groupement archéologique de Seine-et-Marne (Mémoires archéologiques de Seineet-Marne, 1), $234 \mathrm{p}$.

Pilet, Ch., 1980 - La nécropole de Frénouville. Étude d'une population de la fin du IIt à la fin du vIT siècle, Oxford, Hadrian Books (BAR, International Series, 83), 3 vol. $225+339$ p., 217 pl.

PiLET, Ch., 1987 - La Normandie des Grandes Invasions, fin du IIt -fin du VII siècle. Apport de l'archéologie funéraire, Caen, CRDP, 23 p.

Pilet, Ch., Alduc-Le Bagousse, A., BuChet, L. et al., 1994 - La nécropole de Saint-Martin-de-Fontenay. Recherches sur le peuplement de la plaine de Caen du ve siècle av. J.-C. au vIr siècle apr. J.-C., Paris, CNRS Éditions (54e suppl. à Gallia), 560 p., 139 fig., $128 \mathrm{pl}$.

Rabeisen, E., 1990 - La production d'équipement de cavalerie $\mathrm{au} \mathrm{i}^{\mathrm{er}}$ siècle apr. J.-C. à Alesia (Alise-Sainte-Reine, Côte-d'Or, France), Journal of Roman Military Equipment Studies, 1, p. 73-98.

Romeuf, A.-M., 2001 - Les Martres-de-Veyre, le quartier artisanal gallo-romain, Lezoux, Cahiers du Centre archéologique de Lezoux (2, t. 1-2), 439 p.

Roussel, L. (dir.), 1979 - Le mobilier de Mâlain-Mediolanum 1968-1978, Cahier du Mémontois, 4, 115 p., 92 pl.

SIEVERS, S., 1996 - Armes celtiques, germaniques et romaines : ce que nous apprennent les fouilles d'Alésia, dans RedDÉ, M., (dir.), L'armée romaine en Gaule, Paris, Errance, p. 74-75. 
